\title{
The role of phosphatidylserine on the membrane in immunity and blood coagulation
}

\author{
Jiao Wang ${ }^{1 *}$, Changxin Yu', Junyi Zhuang ${ }^{1}$, Wenxin Qi ${ }^{1}$, Jiawen Jiang ${ }^{1}, X^{1}$ uanting Liu', Wanwei Zhao ${ }^{1}$, Yiyang Cao ${ }^{1}$,
} Hao $\mathrm{Wu}^{1}$, Jingxuan $\mathrm{Qi}^{1}$ and Robert Chunhua Zhao ${ }^{1,2,3,4^{*}}$ (i)

\begin{abstract}
The negatively charged aminophospholipid, phosphatidylserine (PtdSer), is located in the inner leaflet of the plasma membrane in normal cells, and may be exposed to the outer leaflet under some immune and blood coagulation processes. Meanwhile, Ptdser exposed to apoptotic cells can be recognized and eliminated by various immune cells, whereas on the surface of activated platelets Ptdser interacts with coagulation factors prompting enhanced production of thrombin which significantly facilitates blood coagulation. In the case where PtdSer fails in exposure or mistakenly occurs, there are occurrences of certain immunological and haematological diseases, such as the Scott syndrome and Systemic lupus erythematosus. Besides, viruses (e.g., Human Immunodeficiency Virus (HIV), Ebola virus (EBOV)) can invade host cells through binding the exposed PtdSer. Most recently, the Corona Virus Disease 2019 (COVID-19) has been similarly linked to PtdSer or its receptors. Therefore, it is essential to comprehensively understand PtdSer and its functional characteristics. Therefore, this review summarizes Ptdser, its eversion mechanism; interaction mechanism, particularly with its immune receptors and coagulation factors; recognition sites; and its function in immune and blood processes. This review illustrates the potential aspects for the underlying pathogenic mechanism of PtdSer-related diseases, and the discovery of new therapeutic strategies as well.
\end{abstract}

Keywords: Phosphatidylserine, GLA domain, Discoidin-like C2 domain, IgV-like domain, TAM, TIM, Blood coagulation (hemostasis), Immunity, Apoptosis, COVID-19

\section{Background}

In eukaryotic cells, the distribution of lipids on biological membranes is asymmetric [1]. Glycosphingolipid, sphingomyelin, and phosphatidylcholine (PtdCho) mainly distribute on the outer leaflet, while aminophospholipids such as phosphatidylethanolamine (PtdEtn) and phosphatidylserine (PtdSer) mostly distribute on the inner leaflet. PtdSer, as an aminophospholipid, its asymmetry distribution on membranes is essential for various biological processes [2]. As

\footnotetext{
* Correspondence: jo717@shu.edu.cn; zhaochunhua@vip.163.com 'School of Life Sciences, Shanghai University, 99 Shangda Road, Shanghai 200444, China

Full list of author information is available at the end of the article
}

Immune and blood coagulation play a vital role in the human body and within them, PtdSer is essential for the normal execution of specific immune and coagulation processes. Once the functions of PtdSer are abnormal, they will cause many diseases, including Scott syndrome [3], Systemic lupus erythematosus (SLE) [4], Essential hypertension (EH) [5], Hemophilia A [6], Alzheimer's disease (AD) [7], Human Immunodeficiency Virus (HIV) [8], Ebola virus (EBOV) [9-12], Dengue virus (DENV) [13-15] and Respiratory Syncytial Virus (RSV) [16-18], and certain tumors or cancers [19-22]. It was also speculated that PtdSer might be a potential mechanism or participant of inflammation and coagulation abnormalities in COVID-19 [23, 24]. 
Therefore, studies in Ptdser, its interacted molecules and their structure features, are of significance, not only an aspect to further understand these diseases but also a potential therapeutic target. Additionally, miles of researches have focused on blood coagulation and inflammation to elucidate the close relationship between them. Molecules that affect blood coagulation usually affect inflammation, and vice versa [25-27]. The Receptor tyrosine kinases TAM family, protein S (PROS1), GLA domain, and C2 domain, binding to PtdSer, all participate in both blood coagulation and immune processes, which means a crucial role of Ptdser in the immune and blood coagulation system (Fig. 1). Therefore, this review focuses on the mechanism of PtdSer exposure during immunity and blood coagulation and how PtdSer interacts with immune receptors and coagulation factors. Meanwhile, this review as well as summarized some PtdSer-related diseases of coagulation or immunity, including COVID-19, in recent years.

\section{The mechanism of PtdSer exposure on apoptotic cells in the immune system}

Apoptosis [36] is also called programmed death, which is the process of cell death caused by the internal and external factors triggering the death program in the cell. There are two main signaling pathways at the beginning, one is the endogenous apoptosis pathway, which is regulated by mitochondria [37]; the other is the exogenous apoptosis pathway, which is regulated by death receptors
[38]. Once apoptosis is activated, cross-talking occurs in these two signal pathways [39], and eventually, they will activate the caspase family proteins (cysteine-containing aspartate-specific proteases). These caspase family members are the executors of apoptosis, resulting in PtdSer exposure in the cell membrane during the pre-apoptotic stage [40-44], which ultimately lead to DNA fracture and protein degradation $[45,46]$. Phagocytes, recognizing PtdSer on the surface of apoptotic cells by its receptors $[19,35,47,48]$, subsequently, engulf the apoptotic cells to avoid inflammation [49].

In normal cells, PtdSer is maintained in the plasma membrane's inner leaflets by flippases such as ATP11A and ATP11C [42]. However, during immune and coagulation processes, ATP11A and ATP11C can be inactivated by other factors like $\mathrm{Ca}^{2+}$ or caspase [41, 42] Simultaneously, scramblase, such as Xk-related Protein 8 (Xkr8) and Transmembrane protein 16F (TMEM16F), will be activated by the same factors, causing PtdSer exposure to the plasma membrane's outer leaflets $[40,50]$.

P4-ATPases, belong to flippase, at least fourteen are encoded in mammals [51-54] and play a crucial role in establishing and maintaining phospholipid asymmetry across the membranes. They transport phospholipids across the membranes rely on ATP hydrolysis particularly when PtdSer and PtdEtn are substrates of P4ATPase [55]. CDC50 family proteins are essential for P4-ATPase to transfer PtdSer from the plasma

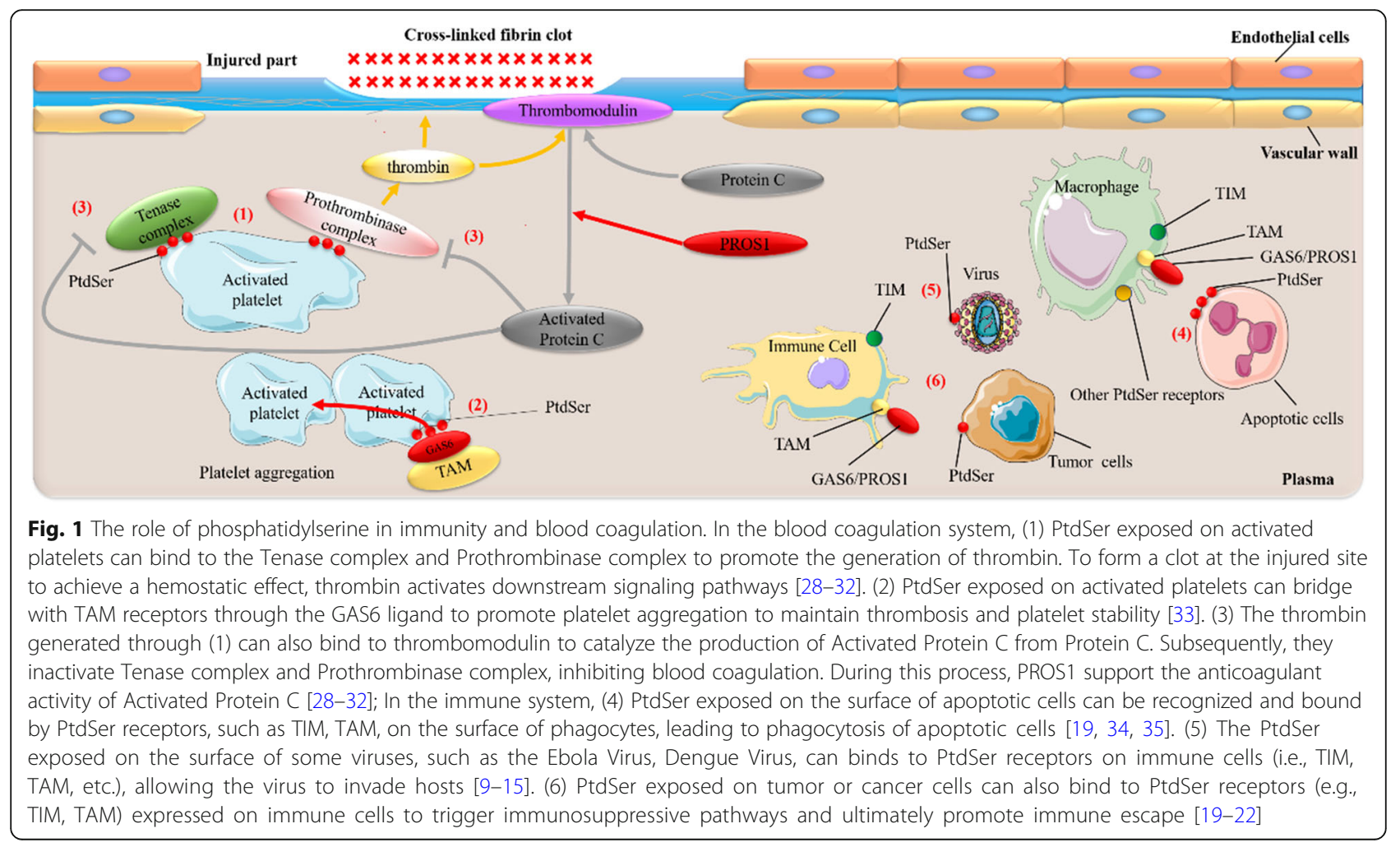


membrane's outer leaflet to the inner leaflet [42]. ATP11C and ATP11A are identified as flippase on the plasma membrane $[41,42] . \mathrm{Ca}^{2+}$ is required to inhibit the activity of flippase (ATP11C and ATP11A) for PtdSer exposure on activated platelets and the cleaved caspase is so for PtdSer exposure on apoptotic cells, and thereby inhibiting PtdSer inversion [41, 42].

Scramblase controls the disruption of lipid asymmetry, catalyzing the non-ATP-dependent bidirectional movement of lipids on the plasma membrane of eukaryotic cells [56]. It includes two protein groups, one is the caspase-dependent Xk family protein [40], and the other is the $\mathrm{Ca}^{2+}$-dependent transmembrane protein 16 (TMEM16) family protein [50]. In eukaryotic cells, two proteins, Xkr8 and TMEM16F, are involved in PtdSer exposure, for which $\mathrm{Xkr} 8$ is responsible on the surface of apoptotic cells, while TMEM16F is on the surface of activated platelets $[40,50]$.

\section{Caspase activates the activity of Xkr8 and inactivates ATP11C and ATP11A}

A large amount of PtdSer is exposed to the apoptotic cell membrane [57]. Once entering the apoptotic or necrosis state [58], apoptotic cells undergo specific surface changes to induce professional or non-professional phagocytes to bind to and then engulf them [58]. The most remarkable surface change is the surface exposure of PtdSer, caused by the loss of plasma membrane asymmetry, in which scramblase Xkr8, caspase 3/7, ATP11A, ATP11C, and CDC50A play a synergistic role (Fig. 2), [40-44].

$\mathrm{Xkr8}$, containing 6 transmembrane regions, is ubiquitously expressed in various tissues and, as a scramblase, is essential for the eversion of PtdSer during apoptosis [40, 44]. Xkr8 has a conservative caspase recognition site in its $\mathrm{C}$-terminal tail region that can be cleaved by caspase $3 / 7$, which is essential for activating its scramblase activity [40]. After Xkr8 is cleaved and activated by caspase, it aggregates with basigin (BSG) or neuroplastin (NPTN) in Ig superfamily. This kind of polymerization is formed by two Xkr8 molecules and two BSG or NPTN molecules to form a heterotetramer, which together complete the function of scramblase for phospholipid scrambling [43].

Caspase can inactivate ATP11A and ATP11C as well. According to cryo-electron microscopy (Cryo-EM), they contain ten transmembrane regions [59]. These phospholipid flippases contain caspase recognition sites, three cleavage sites in the "N" domain (Nucleotide-binding domain) of ATP11C [42], and two cleavage sites in the "P" (phosphorylation domain) and "N" domains of ATP11A [41]. Once these sites are mutated, ATP11C and ATP11A cannot activate their flippase activity. In the apoptosis process, these sites of ATP11C and ATP11A are cleaved by caspase $3 / 7$, to inactivate ATP11C and ATP11A [42], finally leading to PtdSer exposure. This caspase-mediated flippase inactivation is necessary for PtdSer exposure on apoptotic cells [41, 42].



Fig. 2 The process of phosphatidylserine exposure on the surface of apoptotic cells. This figure schematically shows the structure of caspasedependent scramblase Xkr8 and flippase (ATP11A /ATP11C related to CDC50A). When cell apoptosis occurs, caspase 3 or caspase 7 cleaves Xkr8 to activate its scramblase activity $[20,21]$. Meanwhile, caspase 3 or caspase 7 cleaves ATP11A and ATP11C to inactivate their flippase activity, resulting in the loss of lipid asymmetry and PtdSer exposure. Then the macrophages will engulf the apoptotic cells. This form of PtdSer exposure is irreversible [22] 


\section{Diseases caused by reduced PtdSer exposure on the surface of apoptotic cells}

Lupus-like autoimmune disease (or systemic lupus erythematosus (SLE)) is a typical example [4]. The patients' immune system produces autoantibodies to attack their own cells and tissues, causing inflammation and organ damage [60]. The study of Mahiru Kawano [4] found that Xkr8 deficiency severely delayed PtdSer exposure on the surface of apoptotic splenocytes, neutrophils, and thymocytes. The exocytosis of these cells is suppressed. This study shows that Xkr8-mediated PtdSer exposure in apoptotic lymphocytes and senescent neutrophils is a key step in apoptosis. After PtdSer exposure, these cells are engulfed, preventing the release of toxic substances from dying or senescent cells. Once Xkr8 deficiency results in reduced PtdSer exposure, it will activate the immune system, leading to SLE [4].

\section{Immune receptors for PtdSer}

Macrophages only engulf apoptotic cells, but not healthy cells [61]. This specificity depends on the "eat-me" signal on the surface of apoptotic cells [62]. The most representative signal is Phosphatidylserine (PtdSer) signal, the main "eat-me" signal [19]. Due to PtdSer exposure, apoptotic cells can be quickly and effectively recognized and eliminated by phagocytes. On the contrary, once the clearance fails, the apoptotic cells may enter the secondary necrotic stage, cause the phagocytes, and release proinflammatory cytokines and trigger inflammation [49].

There are multiple PtdSer receptors on immune cells. The mechanism for recognition of PtdSer by its receptor is crucial for the the process of endocytosis of immune cells. PtdSer receptor families are multiple, of which two types have been identified as PtdSer-sensing receptors TAM receptor protein tyrosine kinases family (TYRO3, AXL, and MER) and TIM family (T cell/transmembrane, immunoglobulin, and mucin) [35, 47, 48]. Additionally other receptors on immune cells include $\alpha v \beta 3$ or $\alpha v \beta 5$ integrins [63, 64], CD300a [14, 65], BAI1 [66], Stabilin [67, 68], RAGE [69], LOX-1 [70], etc. Most of them all can recognize PtdSer exposure on the surface of apoptotic cells, and thus endocytosis, while some of the downstream mechanisms are still unknown.

\section{TAM receptors family recognizes $\mathrm{PtdSer}$ in the immune and blood coagulation systems}

TAM receptors include TYRO3, AXL, and MER, which are receptor protein tyrosine kinases (RPTKs or RTKs) on the cell surface [71]. RTKs are transmembrane glycoproteins, serve as both receptors and enzymes, binding to ligands and phosphorylate tyrosine residues of target proteins. RTKs binding to homologous ligands form dimers or tetramers, thereby catalyzing the receptor autophosphorylation and tyrosine phosphorylation of the substrates of RTKs. It transmits extracellular signals to the cytoplasm and activates many signal transduction pathways in the cell $[72,73]$.

TAM-RTKs (hereafter referred to as TAM) are members of the RTKs family. They are expressed by many cells, such as Macrophages [74], Dendritic Cells [74, 75], Antigen presenting cell [74], immature natural killer (NK) cells [76], Cerebellar Purkinje cells [77], Hippocampal dentate gyrus [77], Retinal pigment epithelium (RPE) cells [78], and Sertoli cells [79] and play a vital role in hemostasis and anti-inflammatory $[25,80,81]$.

\section{TAM, GAS6/PROS1 and PtdSer in immune system}

The immune system uses many methods to eliminate inflammation, one of which is to activate TAM for antiinflammation. Only when TAM correctly recognizes and binds to PtdSer (Fig. 3) can it avoid inflammation caused by the failure to clear apoptotic cells, then play the antiinflammatory effect of TAM [34, 49]. TAM does not directly bind to the PtdSer exposed on the cell surface but relies on growth-arrest-specific 6 (GAS6) or protein S (PROS1) as a ligand [82]. Both GAS6 and PROS1 are vitamin K-dependent proteins with similar structures (Fig. 3). Their C-terminal is used for TAM binding and phosphorylation to be used as TAM ligands [83]. Studies have found that the binding of GAS6/PROS1 to TAM does not depend on PtdSer and $\mathrm{Ca}^{2+}$. The optimal activation of TAM receptors for any ligand requires the presence of PtdSer and $\mathrm{Ca}^{2+}$ that bind to the GLA domain of the ligand. In the signal transduction process, PtdSer, $\gamma$-carboxylated GAS6/PROS1 ligand, and TAM receptor together constitute a complete TAM signal transduction module [84].

It is worth noting that GAS6 binds and activates all three receptors, while PROS1 binds and activates TYRO3 and MER but does not activate AXL [84]. Through receptor-ligand activation curves, the difference in TAM signal is from the two N-terminal Ig-like domains of the TAM receptor but not the difference in TAM kinase activity [84]. Most or all of GAS6 in cells and tissues in the body have specifically bound to AXL, showing AXL dependence. The affinity of GAS6: AXL $\geq$ TYRO3 >> MER [84].

After binding to PtdSer, TAM activates downstream signaling pathways:

(i) TAM receptors expressed on macrophages or dendritic cells can recognize PtdSer on the surface of apoptotic cells and activate phagocytosis by activating Rac1 (a GTPase) [85]. Rac1 is a member of Ras Homologue (Rho) GTPase. Rho GTPase mainly affects the remodeling of the cytoskeleton. Rac1 acts as a positive regulator of phagocytosis by inducing cytoskeleton rearrangement [80, 85-87]. 


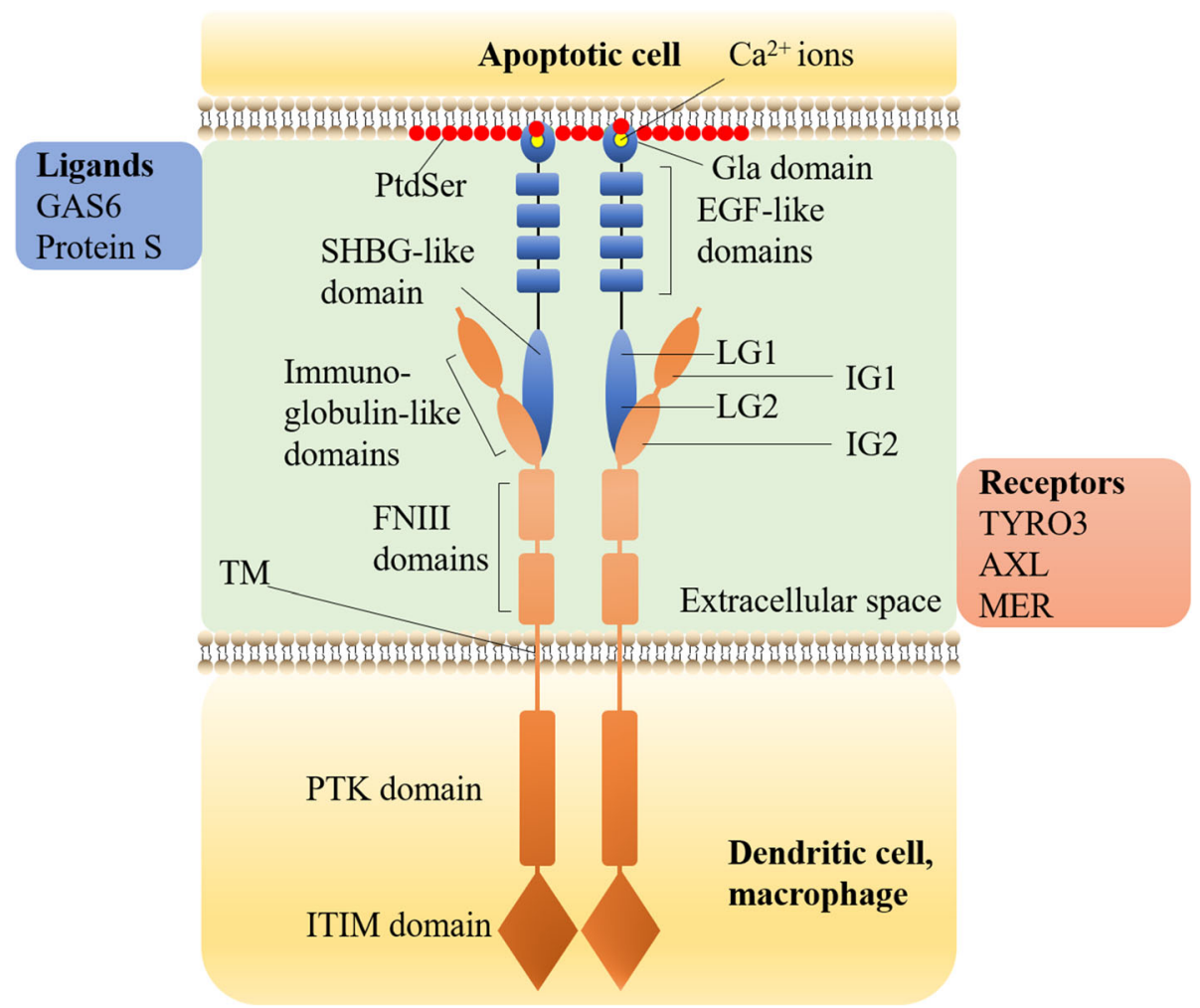

Fig. 3 The Structure of TAM, GAS6/Protein S and how they bind to PtdSer. TAM is a single transmembrane receptor (the orange part in the figure). From N-terminal to C-terminal (from top to bottom in the figure), there are three parts:1, the extracellular domain, including two IG domains (IG1, IG2) and two FNIII domains; 2, one single TM; 3, the intracellular domain, including one conserved PTK domain, one autophosphorylation site, and one ITIM domain [23-27]. The intracellular PTK domain of TAM is the functional domain. TYRO3, AXL, and MER of TAM family all have phosphorylation and autophosphorylation sites, which can activate the corresponding signaling pathways by phosphorylation of downstream target proteins. TAM does not directly bind to the PtdSer exposed on the cell surface but relies on GAS6 or PROS1 as a ligand [28]. GAS6/ PROS1 consists of three parts (the blue part in the figure). From N-terminal to C-terminal (from top to bottom in the figure), there are $\mathrm{N}$-terminal GLA domain, four EH -like domains, and C-terminal SHBG domain [7, 29, 30]. The TAM receptor binds to the C-terminal SHBG domain of GAS6/ PROS1 through two N-terminal IG1 and IG2 in the extracellular domain. SHBG domain includes two tandem LG domains (LG1, LG2). The binding eventually leads to the activation of TAM receptor tyrosine kinase. GAS6/PROS1 binds to the PtdSer exposed outside the cell through the Nterminal GLA domain. With the assistance of vitamin K, the structure of the GLA domain can be stabilized through the binding of about 7 essential $\mathrm{Ca}^{2+}$ ions (yellow ball at the top in the figure) [7, 31-34]. The specific mechanism of interaction between the GLA domain and PtdSer will be introduced in section 4.2

(ii) TAM receptor on immune cells and GAS6/PROS1 ligand binding to PtdSer on apoptotic cells have direct anti-inflammatory activity that suppresses inflammatory cytokines and nuclear factor- $\mathrm{kB}$ (NFкB) $[19,88]$. For example, TAM prevents inflammation in our body by inhibiting the Toll-like receptor (TLR) and TLR-induced cytokine receptor cascade [75].. It has been shown that TLR signaling significantly reduces the expression of GAS6 in mouse macrophages by activating NF- $\kappa B$, thereby further promoting TLR-mediated inflammation in a selfregulating manner [89]. Researches have shown that the MER receptor expressed on macrophages and dendritic cells has an inhibitory effect on the activation of NF-kB. This effect is MER-specific [90]. Also, GAS6-induced AXL activation inhibits TLR and type I interferon (IFN) receptor signal transduction by up-regulating the expression of SOCS1 and SOCS3 (Suppressor of cytokine signaling (SOCS) protein can inhibit the activity of TLR), thereby shutting down the expression of inflammatory cytokines including Tumor necrosis factor (TNF)- $\alpha$, IFN- $\alpha$, Interleukin (IL)- $1 \beta[19,75]$.

(iii) The pathway to clear apoptotic cells also involves phosphatidylinositol 3-kinases (PI3K), phospholipase $\mathrm{C} \gamma 2$, Src family kinases, and interactions with the $\alpha \mathrm{v} \beta 5$ integrin, etc.

TAM is a single transmembrane receptor (the orange part in the figure). From $\mathrm{N}$-terminal to $\mathrm{C}$-terminal (from top to bottom in the figure), there are three parts:1, the extracellular domain, including two IG domains (IG1, 
IG2) and two FNIII domains; 2, one single TM; 3, the intracellular domain, including one conserved PTK domain, one autophosphorylation site, and one ITIM domain [80, 91-94]. The intracellular PTK domain of TAM is the functional domain. TYRO3, AXL, and MER of TAM family all have phosphorylation and autophosphorylation sites, which can activate the corresponding signaling pathways by phosphorylation of downstream target proteins.

TAM does not directly bind to the PtdSer exposed on the cell surface but relies on GAS6 or PROS1 as a ligand [82]. GAS6/PROS1 consists of three parts (the blue part in the figure). From N-terminal to C-terminal (from top to bottom in the figure), there are N-terminal GLA domain, four EH -like domains, and C-terminal SHBG domain $[34,95,96]$.

The TAM receptor binds to the C-terminal SHBG domain of GAS6/PROS1 through two N-terminal IG1 and IG2 in the extracellular domain. SHBG domain includes two tandem LG domains (LG1, LG2). The binding eventually leads to the activation of TAM receptor tyrosine kinase. GAS6/PROS1 binds to the PtdSer exposed outside the cell through the N-terminal GLA domain. With the assistance of vitamin $\mathrm{K}$, the structure of the GLA domain can be stabilized through the binding of about 7 essential $\mathrm{Ca}^{2+}$ ions (yellow ball at the top in the figure) $[34,97-100]$. The specific mechanism of interaction between the GLA domain and PtdSer will be introduced in section Vitamin K-dependent coagulation factors bind to PtdSer.

\section{TAM, GAS6/PROS1 and PtdSer in blood coagulation system} When GAS6 ligands interact with TAM receptor family members (TYRO3, AXL, and MER) under hemostatic conditions, they can promote platelet aggregation to maintain thrombosis and platelet stability $[25,33,81,101$, 102]. During this process, PtdSer will be exposed to activated platelets to participate in the production of thrombin [103] and will also bind to GAS6 to activate TAM [33]. PROS1 can be used as an anticoagulant in the anticoagulation process of Activated Protein C (APC) to inhibit coagulation. This anticoagulation process does not require the participation of TAM but must involve the participation of PtdSer (section The Cell-Based Model of Hemostasis involving PtdSer and Application of PtdSer in blood coagulation in this review). In addition, GAS6 was not found to be involved in the anticoagulation process of APC [104-107].

\section{TAM and PtdSer in disease}

Some diseases and pathological processes may involve the binding of TAM to PtdSer. For example, AD [7], Tumor and cancer such as breast cancer [22], DENV [15], EBOV [10-12], and RSV [16-18] are involved.
In $\mathrm{AD}$, neuroinflammation stimulates microglia to produce superoxide, release nitric oxide, and then superoxide, which induces PtdSer exposure in neurons [7]. Furthermore, when exposed to soluble $A \beta$ oligomers, microglia in adult brains engulf synaptic material through the CR3dependent process [108]. These findings indicate that the PtdSer-exposed synaptic compartment can further result in excessive phagocytosis through the GAS6/PROS1/ TAM receptor pathway and cause synapse loss in AD [109]. It may aggravate the symptoms of dementia such as aphasia, memory impairment, executive dysfunction, and behavior changes in $\mathrm{AD}$ patients.

As mentioned above, TAM receptors binding to PtdSer-exposed apoptotic cells by binding to the ligands GAS6/ PROS1 has a function of regulating immunity. In cancer, PtdSer is often exposed on the surface of tumor cells, tumor vascular endothelial cells, and tumor-derived vesicles [110-112]. At the same time, it is worth noting that the TAM receptor and their two ligands, GAS6/ PROS1, have been reported to abnormally express in various tumor microenvironments or promote tumor growth and infiltration [19, 47, 113-117]. It hints that the TAM receptor may play an immunological role in the tumor microenvironment through its ligand and PtdSer-exposed cells. Besides, Canan Kasikara et al., using TAM-IFN $\gamma \mathrm{R} 1$ reporter lines and expressing TAM receptors in various epithelial cell models, revealed PtdSer regulates PD-L1 expression via TAM receptor, which fosters immune evasion and chemoresistance [118]. It indicates that PtdSer participates in the immune examination process in cancer through binging to the TAM receptor. In short, more researches are needed to illustrate the potential functions of the PtdSer, TAM receptors, and their ligands GAS6/ PROS1 in cancer.

Some viruses, such as Dengue [15], EBOV [10-12], RSV [16-18], can use multiple PtdSer receptors (e.g., TAM receptors) in our body to promote their attachment, entry, and replication in host cells through the interaction of bridge proteins such as GAS6/PROS1 with PtdSer [119].

\section{TIM receptors family recognizes PtdSer in the immune system}

The human TIM family includes three TIM proteins, including TIM-1, TIM-3, and TIM-4. They share a common structure, Immunoglobulin variable (IgV)like domain that allows them to recognize PtdSer exposed on the surface of apoptotic cells with a high degree of specificity [35] (Fig. 4). TIM family regulates immune responses, including autoimmunity, transplant tolerance, the response to viral infections, and the regulation of allergy and asthma [120, 121]. 




Human TIM-1 (hTIM-1) is related to allergies, asthma, and autoimmune diseases, which indicates that TIM-1 regulates the immune system more comprehensively [35]. mTIM-1 (mouse TIM-1) is preferentially expressed in $\mathrm{T}$ helper 2 (Th2) cells, transmits signals that enhance $\mathrm{T}$ cell activation and proliferation, and increases airway hyper-reactivity and allergic reaction [122].

Tim-3 was originally thought to be expressed on mouse $\mathrm{T}$ helper 1 (Th1) and T helper 17 (Th17) cells. Also, cell subsets in the innate immune system express TIM-3, including human natural killer cells, monocytes [123] and dendritic cells [124], macrophages [125], and dendritic cells [126].
TIM-4 does not express in $\mathrm{T}$ cells. It expresses on antigen-presenting cells, such as monocytes-phagocytes, dendritic cells, B cells [127], iNKT cells [128], and even tumor cells [129].

\section{TIM binds to PtdSer}

Cells expressing TIM family members bind to or phagocytose apoptotic cells expressing PtdSer (Fig. 4 a) [127, 132, 133, 137].TIM-3 has various ligands, such as PtdSer and Galectin-9 (Fig. 4 c) [135]. Galectin-9 is an S-type lectin, which is a ligand of TIM-3. Galectin-9 binds to the carbohydrate chain on TIM-3, induces T helper 1 (Th1) cell death [135], and acts as a negative regulator of Th1 / $\mathrm{T}$ cytotoxic cell (Tc1) function [138]. The 
interaction between TIM-3 and PtdSer does not exclude the interaction with Galectin-9, because the binding site is located on the opposite side of the IgV domain [35]. The binding of PtdSer and TIM-3 phosphorylates tyrosine residues in the cytoplasmic tail of TIM-3. The binding is essential for TIM-3 signaling, but the downstream signaling mechanism in T cells is still unclear $[139,140]$. It hints that the interaction of TIM-3 and PtdSer may induce apoptotic cell phagocytosis and antigen crosspresentation [137].

In phagocytes, TIM- 4 can directly bind to PtdSer (Fig. 4 b (2)) [127] or indirectly through exosomes (Fig. 4 b (1)) [134]. Macrophages expressing TIM-4 recognize PtdSer of apoptotic cells, which leads to phagocytosis and clearance of apoptotic cells. However, the cytoplasmic domain of TIM-4 has only about 40 amino acids, which is too short to contain any tyrosine signaling motifs [35]. TIM-4 is considered to use the activity of TAM tyrosine kinase for signal transduction. TIM-4 alone cannot support phagocytose apoptotic cells but can significantly enhance the endocytosis mediated by the TAM signal system [141]. However, in the process of TIM-4 mediated endocytosis, the molecular mechanism of TIM-4 signal transduction to phagocytes is not fully understood to date. A recent study found that the physical interaction between TIM-4 and MER is necessary for MER to enhance TIM- 4 mediated endocytosis. This physical interaction is mediated by the interaction between the $\operatorname{IgV}$ domain of TIM- 4 and the fibronectin-like III domain (FNIII domain) of MER [142], which partially explains the mechanism above. Furthermore, TIM-4 is also considered to be the ligand of TIM-1. TIM-4 and TIM-1 can bind to each other with PtdSer on the Exosome to form a bridge. TIM-4 and TIM- 1 may participate in cell-cell interactions through PtdSer on exosomes [134].

Additionally, exosomes expose PtdSer [143] on their outer leaflet and contain various cellular proteins, including galectins [144]. These extracellular vesicles are thought to be involved in many biological processes such as immune response and be involved in the communication between cells [145]. TIM protein may become one of the receptors of exosomes through PtdSer. Exosomes may bridge any two TIM proteins except TIM-2 (Fig. 4 b (1)) [134].

\section{TIM and PtdSer in immune system}

The binding of TIM family to PtdSer is a critical signal in the immune system. TIM-1 is constitutively expressed in Invariant natural killer $\mathrm{T}$ (iNKT) cells. Its recognition of PtdSer can induce iNKT cell activation, proliferation, and cytokine production [146]. At the same time, TIM-1 can also be highly upregulated on the surface of damaged renal epithelial cells, then giving epithelial cells the characteristics of semi-professional phagocytes. Apoptotic substances in the renal tubules can be removed by identifying and binding PtdSer on the surface of apoptotic cells [147]. Since TIM-1 can be expressed in injured and cancerous kidney samples, it is also known as kidney-injury-molecule-1 (KIM-1) in kidney and oncology [148]. So far, the role of PtdSer in regulating the function of TIM-3 is unclear. Studies have shown that the interaction between TIM-3 and PtdSer may participate in the phagocytosis of apoptotic cells and the subsequent cross-presentation of antigens [137]. TIM-4 on macrophages can bind to PtdSer on the surface of apoptotic cells to mediate the clearance of apoptotic cells [35]. However, the molecular mechanism by which TIM-4 transmits signals to phagocytes in the process of PtdSer and TIM-4 mediated endocytosis is not yet fully understood. TIM-4 is considered to require the tyrosine kinase activity of MER in the TAM receptor family for signal transduction [141].

\section{TIM and PtdSer in disease}

Many viruses invade cells by using the PtdSer exposed on their surface to interact with PtdSer receptors (such as TIM receptors), including HIV [149], EBOV [9], DENV [13-15], etc.

Acquired immunodeficiency syndrome (AIDS) is an immunodeficiency disease mainly caused by HIV infection [149]. Studies have found that multiple endocytic pathways promote the internalization of HIV-1 into epithelial cells. HIV-1 guides itself into the cell through interaction with PtdSer, TIM-1, heparan sulfate proteoglycans (HSPG), galactosylceramide (GalCer), and endocytosis and macropinocytosis [8]. TIM-1 also promotes HIV-1 entry into CD4+ T lymphocytes, However, the interaction between TIM-1 and PtdSer exposed on the surface of HIV during the release of progeny virions inhibits the release of the virus and retains the virus particles on the cell surface, thereby reducing the production and replication of HIV-1 [150].

EBOV, a member of the Filoviridae family of viruses, utilizes PtdSer receptors for entry into target cells. The PtdSer on the surface of the virus particle mediates the entry of filovirus through the conserved PtdSer binding pocket (MILIBS) in the amino-terminal IgV domain of TIM-1 and TIM-4. This discovery deepens understanding of the interaction between TIM-4 and EBOV virus particles [9].

Dengue is a viral disease transmitted by mosquitoes in tropical and subtropical regions. Studies have shown that TIM-1 can promote virus entry into cells during DENV infection and activate DENV autophagy [13]. DENV simulates apoptotic cells by exposing PtdSer on the viral membrane to promote the binding of TIM-1 [14]. 
In the tumor microenvironment, the interaction of PtdSer (exposed on cancer cells or tumor-derived exosomes) and PtdSer receptors (such as TIM-3 and TAM) expressed on immune cells can trigger a preciselycontrolled-immunosuppressive pathway to weaken the innate and adaptive immune response and ultimately promote immune escape [19-21].

\section{Other immune receptors recognize PtdSer in the immune} system

In addition to the TAM family and TIM family receptors, there are other receptors on the surface of macrophages, dendritic cells, and certain endothelial cells. For instance, $\alpha v \beta 3$ or $\alpha v \beta 5$ integrins [63, 64], CD300a [14, 65], BAI1 [66], Stabilin [67, 68], RAGE [69], LOX-1 [70], etc. They bind to PtdSer in a direct or indirect manner and recognize PtdSer on the surface of apoptotic cells, leading to endocytosis (Table 1).

\section{The mechanism of PtdSer exposure on activated platelets in the blood coagulation system}

Under normal circumstances, platelets isolate the aminophospholipids, PtdSer and PtdEtn, in the inner leaflets of the membrane, while the PtdCho preferentially occupy the outer leaflets of the membrane [176]. This asymmetry is also maintained by flippase (such as ATP11C and ATP11A), which can transport PtdSer and PtdEtn from the outer leaflets of the plasma membrane to the inner leaflets [177]. When the tissue cells are not damaged, the coagulation factor mainly contacts choline phospholipids on the blood cells. These phospholipids constitute a non-thrombogenic surface [178]. Damaged tissue cells activate platelets, therewith the level of $\mathrm{Ca}^{2+}$ in the platelet cytoplasm increases which triggers changes in the lipid composition of the platelet membrane as PtdSer externalization [179]. Once exposed to the surface of platelets, PtdSer binds to various coagulation factors and promotes their enzymatic activity, eventually leading to the massive production of thrombin. The thrombin is indispensable for forming clots in damaged tissue cells, and the resulting clots can achieve hemostatic effects [28-32, 103]. Therefore, the normal exposure of PtdSer is extremely important for the subsequent generation of thrombin, thereby triggering the hemostatic effect.

Increased $\mathrm{Ca}^{2+}$ concentration activates the activity of TMEM16F and inactivates ATP11C and ATP11A

TMEM16F belongs to the TMEM16 family and is a $\mathrm{Ca}^{2+}$-dependent PtdSer scramblase as mentioned above [50]. TMEM16F, an ion channel, is essential to activate $\mathrm{Ca}^{2+}$-dependent externalization of PtdSer in platelets [19, 180, 181] (Fig. 5). It is comprised of ten transmembrane helices (TM). In the absence of $\mathrm{Ca}^{2+}$, its hydrophobic residue F518 of TM4 and hydrophobic residue I612 of TM6 will be close to each other to prevent the phospholipid head group from approaching the hydrophilic cavity; when $\mathrm{Ca}^{2+}$ binds to the hydrophilic cavity, the conformation of the cavity changes, which may cause TM6 to move around the conservative glycine hinge and rearrange TM3, TM4, and TM5, eventually leading to the separation of TM4 and TM6. Subsequently, the inside of the hydrophilic cavity is exposed to the surrounding phospholipids, and the phospholipid head group can enter the cavity, thereby activating TMEM16F [182]. In summary, the mechanism of $\mathrm{Ca}^{2+}$ activating TMEM16F is to change the conformation of the hydrophilic cavity of TMEM16F so that the hydrophilic cavity can be exposed to bind to the phospholipid head group. In the absence of $\mathrm{Ca}^{2+}$, the hydrophilic cavity will be closed by residues on the protein, preventing the hydrophilic cavity from binding to the phospholipid head group. Scott syndrome is a rare bleeding disorder causing increased epistaxis after trauma, often occurring after tooth extraction, and causing severe Postpartum hemorrhage [183]. Studies revealed it caused by insufficient $\mathrm{Ca}^{2+}$-dependent PtdSer exposure in activated platelets and mutations in the TMEM16F gene can cause it as well $[3,50]$.

Inactivation of ATP11C is also a $\mathrm{Ca}^{2+}$-mediated manner, which induced by activation of $\mathrm{Ca}^{2+}$-dependent protein kinase C (PKC $\alpha)$ [185]. PKC phosphorylates Ser1116, in the C-terminal cytoplasmic region of ATP11C, which leads to the production of the di-leucine sequence (pSVRPLL), corresponding to Leu1120 and Leu1121. The resulting pSVRPLL can be used for endocytosis [185]. Adaptor protein complex 2 (AP-2) is a key protein in clathrin-mediated endocytosis [186]. AP-2 can recognize two main endocytosis motifs, one of which is the acidic di-leucine motif $[187,188]$. ATP11C is endocytosed by clathrin-mediated endocytosis, subsequently the flippase activity decreasing [185].

So far, there is no precise mechanism to explain the principle of $\mathrm{Ca}^{2+}$ inactivating ATP11A. However, studies have shown that $\mathrm{Ca}^{2+}$ can indeed inactivate ATP11A in the process of PtdSer exposure on platelets [41, 185]. Although ATP11A, like ATP11C, contains a sequence similar to the dileucine signal in its C-terminal cytoplasmic region, this sequence cannot act as an endocytosis signal through the $\mathrm{Ca}^{2+}$-mediated $\mathrm{PKC}$ activation pathway [185]. Therefore, the mechanism of $\mathrm{Ca}^{2+}$ inactivating ATP11A is not the same as that in ATP11C.

\section{PtdSer involved in the process of blood coagulation and anticoagulation} The cell-based model of hemostasis involving PtdSer The latest cell-based hemostasis model [28-32] replaces the original coagulation cascade model [103] because the 


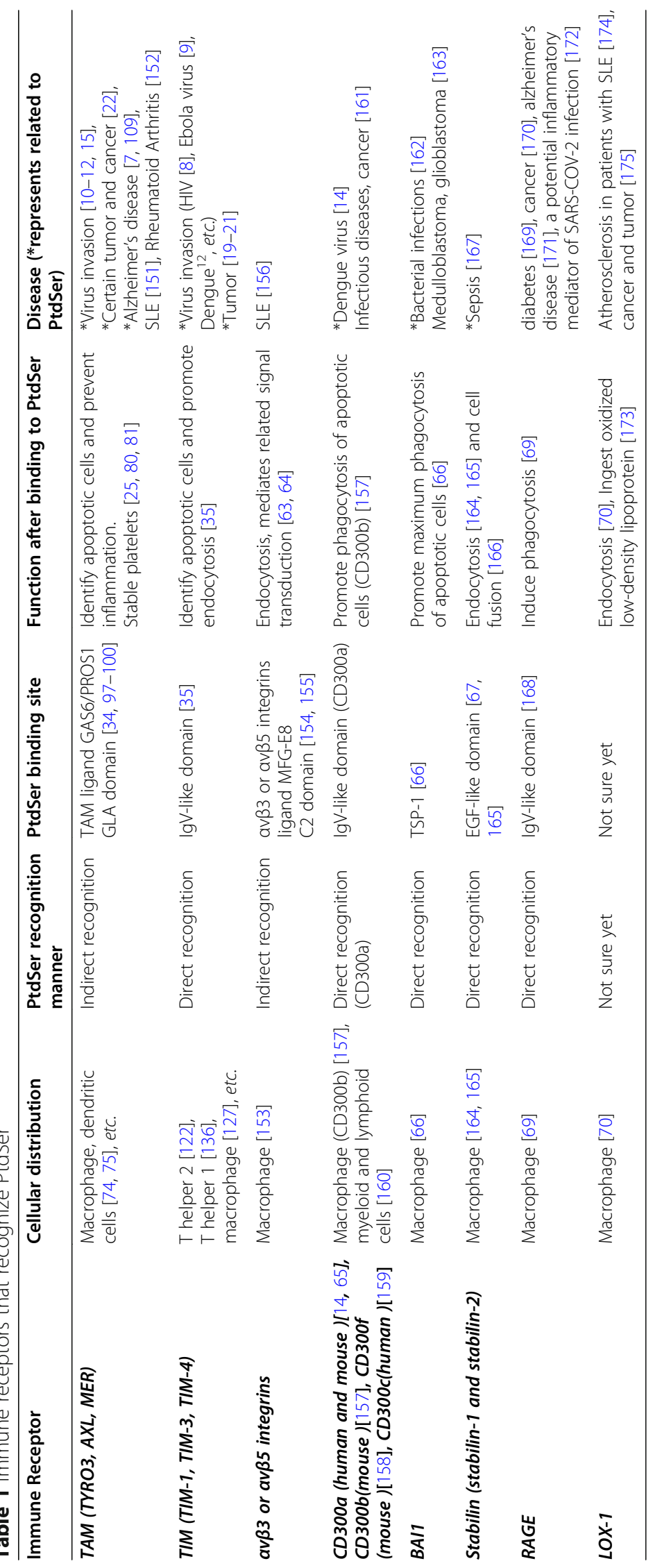




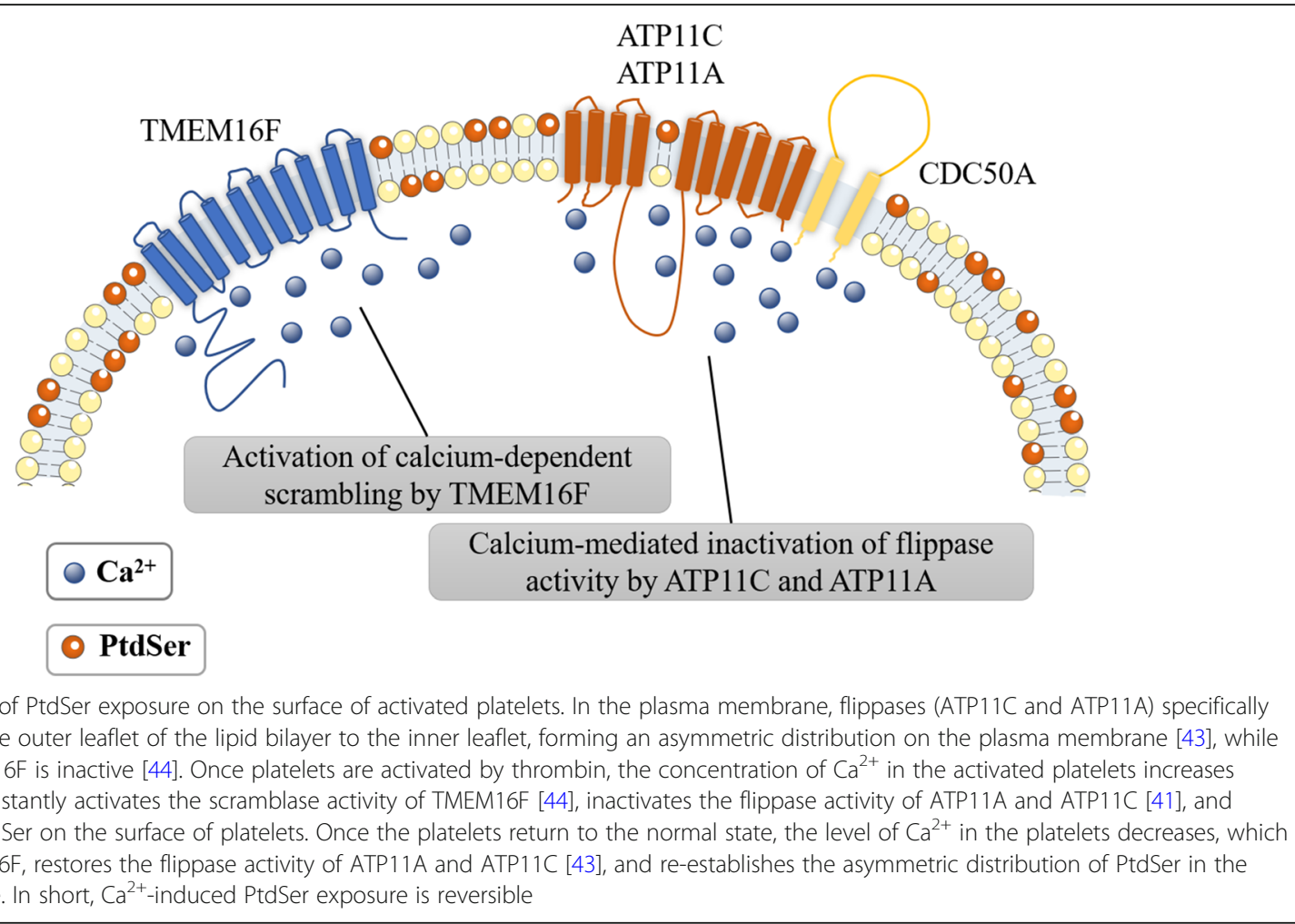

new model can better explain clinical hemostasis in vivo. The cell-based hemostasis model can be divided into three stages, initial stage, amplification stage, and propagation stage (Fig. 6). When the endothelial cells rupture, the tissue factor (TF) on the fibroblasts under the endothelium is exposed to the blood, which triggers the initiation of blood coagulation (Fig. 6 a).

During the coagulation process, after platelet activation, the content of PtdSer on the outer leaflets of platelets increases significantly. Tenase complex and Prothrombinase complex can interact with PtdSer to promote thrombin generation [103]. During the assembly process of Tenase complex (FVIIIa and FIXa) and Prothrombinase complex (FVa and FXa) (Fig. 6 b, c), FVIIIa and FVa act respectively as cofactors of FIXa and FXa [103]. Furthermore, $\mathrm{Ca}^{2+}$ and PtdSer are necessary for the function of these complexes. FIXa and FXa are vitamin K-dependent coagulation factors. They have a GLA domain, which binds to the phospholipids (such as PtdSer) on the platelet membrane in a $\mathrm{Ca}^{2+}$-dependent manner [189]. FVIIIa and FVa also require $\mathrm{Ca}^{2+}$ to stabilize their own connection after activation [190]. The light chains of FVIIIa and FVa bind to phospholipid membranes (such as PtdSer on the membrane), which is necessary to stabilize the protein after activation [191]. However, the binding of PtdSer and discoidin-like C2 domain in their light chain does not need to rely on
$\mathrm{Ca}^{2+}$ [192]. There are three ways of anticoagulation to regulate blood coagulation.

(i) Tissue factor pathway inhibitor (TFPI) is a coagulation inhibitor that can regulate the initiation of coagulation induced by tissue factor (TF) (Fig. 6 a) [193].

(ii) Antithrombin (AT) is an enzyme that inhibits the coagulation system, which is a serine protease inhibitor (serpin) [194]. The physiological role of AT is to protect the circulation from the destruction of released enzymes and limit the coagulation process to the vascular injury site (Fig. $6 \mathrm{a}, \mathrm{c})$. AT itself is an ineffective serine protease inhibitor. Heparin and heparinoid molecules on the surface of endothelial cells will activate it [195].

(iii) Another anticoagulant pathway is the PtdSerdependent APC anticoagulant system. It inhibits the activities of FVIIIa and FVa (Fig. 6 b). In other words, it inhibits the function of the Tenase complex and Prothrombinase complex [196]. Thrombomodulin is a transmembrane protein of endothelial cells and peripheral blood cells, binds thrombin (FIIa) with high affinity, and changes its substrate specificity. The complex formed by thrombomodulin and IIa becomes an effective activator of zymogen protein $\mathrm{C}$, which can activate zymogen protein C to APC [197]. APC can inactivate FVIIIa and FVa 


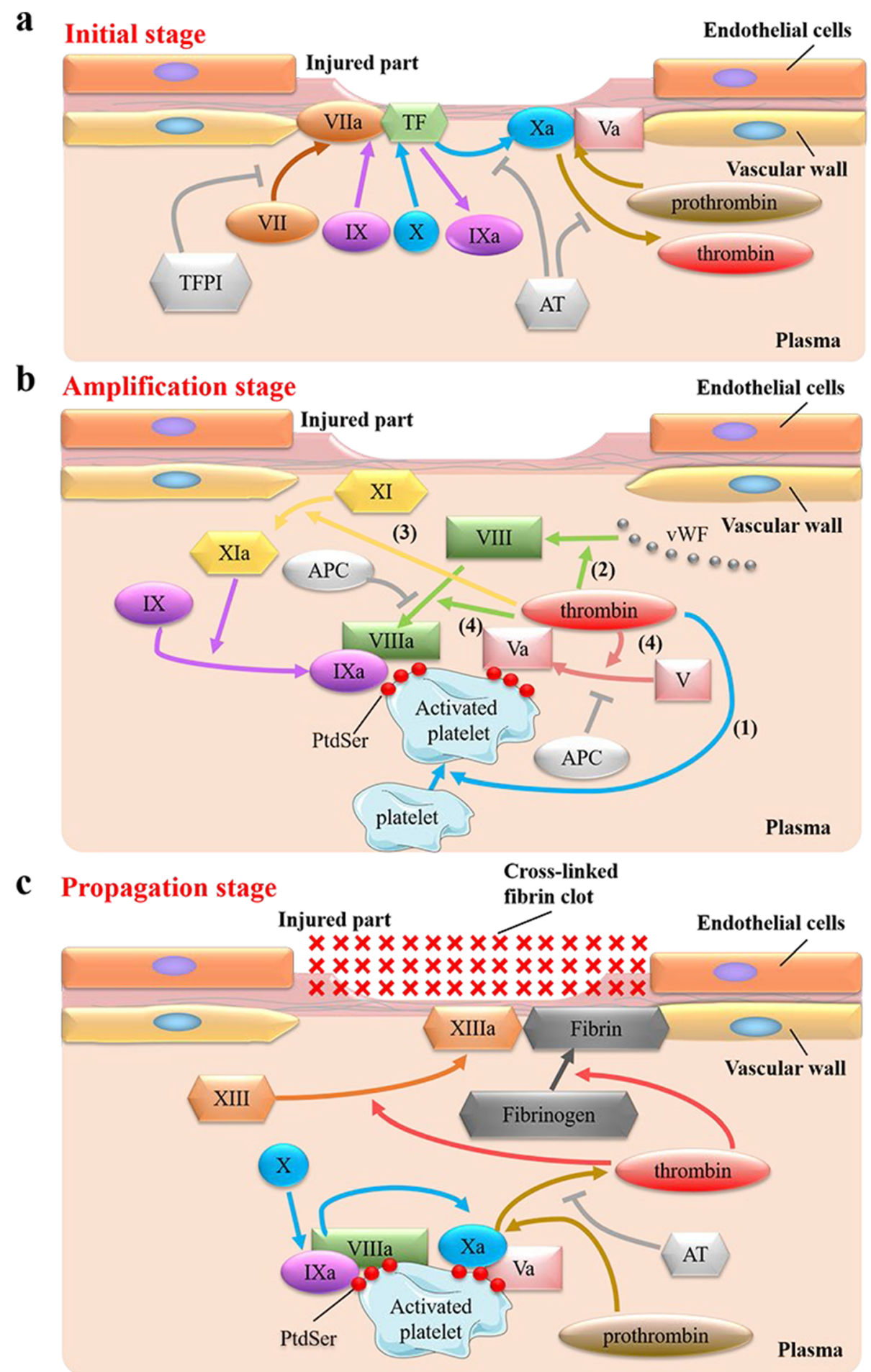

Fig. 6 (See legend on next page.) 
(See figure on previous page.)

Fig. 6 The Cell-Based Model of Hemostasis. a Initial stage of hemostasis. Plasma factor VII (FVII) binds to tissue factor (TF), then FVII automatically activates to FVIla. On the surface of endothelial cells, FVIla and TF together form a complex (FVIla/TF complex). FVIla/TF complex activates Factor IX (FIX) and Factor X (FX) to FIXa and FXa. On the same cell surface, FXa can form a Prothrombinase complex with its cofactor Va (FVa). The Prothrombinase complex can convert prothrombin (FII) to thrombin (Flla). In this process, a small amount of thrombin is produced. However, a small amount of thrombin is not enough for hemostasis, and more thrombin needs to be generated further. $\mathbf{b}$ Amplification stage of hemostasis. The small amount of thrombin just generated has four functions in this process (the number here does not represent the order of reaction). (1) Thrombin activates the platelets in the plasma. The platelets are activated to form a thrombus formation surface. At the same time, PtdSer is exposed to the surface of the activated platelets. (2) Thrombin can also release Factor VIII (FVIII) from its carrier von Willebrand Factor (vWF). (3) Thrombin can activate Factor XI (FXI) to produce FXIa, then FXla can activate Factor IX (FIX) to FIXa. (4) Thrombin activates Factor V (FV) to FVa and activates the newly released FVIII to FVIIIa. In these processes, the activation process of FIXa, FVa, and FVIIla is carried out on the surface of newly activated platelets. At this time, on the surface of activated platelets, FIXa, FVa and FVIIla are bound for the next stage of reaction. $\mathbf{c}$ Propagation stage of hemostasis. On the surface of activated platelets, FVIIla and FIXa form a Tenase complex, which can activate FX to FXa. In addition, the rate at which Tenase complex activates Factor $\mathrm{X}$ to Factor $\mathrm{Xa}$ is faster than FVIla/TF complex. FXa and FVa form a Prothrombinase complex, which allows prothrombin (FII) to be converted to thrombin (Flla), resulting in massive production of thrombin - "thrombin burst". PtdSer can promote the mass production of thrombin by binding with the Tenase complex and Prothrombinase complex. After "thrombin burst", a large amount of thrombin can convert Fibrinogen (FI) to Fibrin (Fla) and can also activate Factor XIII (FXIII) to FXIIla. FXIIla can cross-link with Fla to form a Cross-linked fibrin clot, thereby forming a clot at the injured site to achieve hemostatic effect. Abbreviations: TFPI Tissue factor pathway inhibitor, AT Antithrombin, VWF von Willebrand Factor, APC Activated protein C

binding to PtdSer on activated platelets via a $\mathrm{Ca}^{2+}$ dependent manner, lead to the decomposition and inactivation of the Tenase complex and Prothrombinase complex, and inhibit blood coagulation.

\section{Vitamin K-dependent coagulation factors bind to PtdSer} Proteins FVII, FIX, FX, Prothrombin APC have a GLA domain at the N-terminal, which can bind to PtdSer in a $\mathrm{Ca}^{2+}$-depend manner and participate in the process of coagulation and anticoagulation [189]. In this process, vitamin $K$ is necessary for the post-translational modification of GLA residues as well as the correct folding and activity [198]. So these coagulation factors can also be called vitamin K-dependent coagulation factors [103]. Also, in section TAM receptors family recognizes PtdSer in the immune and blood coagulation systems, it is mentioned that the ligand GAS6/PROS1 of TAM can also recognize and bind to PtdSer through the GLA domain, and GAS6/PROS1 is also vitamin K-dependent [34, 97100].

The binding of the GLA domain to the PtdSerexposed membrane is mediated by multiple interactions: Calcium coordination, ions, van der Waals forces, and hydrophobic interactions [100]. GLA domains from



\section{Factor VIII (330Kda)}

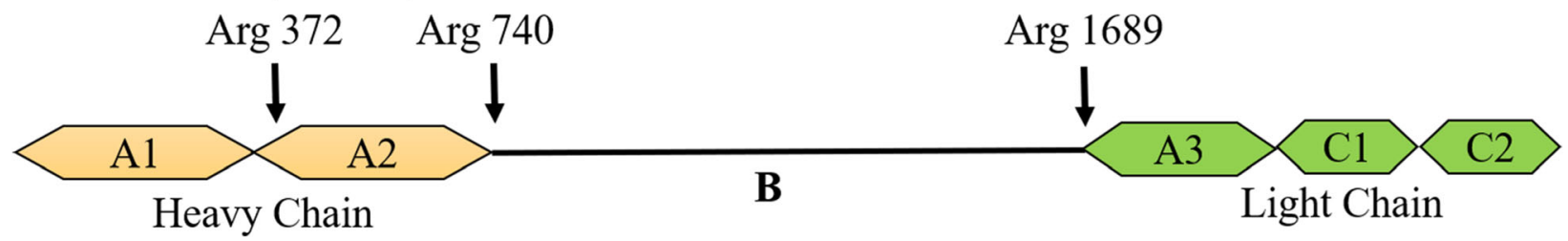

Fig. 7 The structure of Factor $V$ and Factor $V I I I$. The figure indicates the cleavage site of Factor $V$ and Factor $V I I I$. After cleaved, Factor $V$ and Factor VIII convert into their active form.Factor $V$ and Factor VIII have the same sequence homology in their three A domains (A1, A2, A3) and two C domains $(C 1, C 2)$, but entirely different sequence homology in their $B$ domains. Both of them are converted by thrombin into their activated form (Factor Va and Factor VIIla) [46]. Thrombin cuts off the non-homologous B domain, producing a heavy chain (A1-A2) and a light chain (A3C1-C2), which are non-covalently combined into a heterodimer and stably connected by Ca2+ (For Factor VIII, A1 and A2 also need to be cut once more to form a heterotrimer) [47]. Their heavy chains contain the properties of cofactors that promote the activity of the Prothrombin complex and Tenase complex [48]. The light chains bind them to phospholipid membranes (such as PtdSer on the membrane). The light chains are necessary for stabilizing proteins after activation [49] 
different coagulation factors show significant differences in lipid specificity and membrane binding affinity, which may depend on the interaction of non-conserved GLA domain residues of these coagulation factors with PtdSer [100, 103].

The GLA domain's function is highly dependent on $\mathrm{Ca}^{2+}$ ions. Researches have established an atomic model of membrane binding GLA domains [199], showing that $\mathrm{Ca}^{2+}$ ions have two roles in the GLA domain: The four internal $\mathrm{Ca}^{2+}$ ions are mainly responsible for the correct folding of the GLA domain to insert into the membrane; the external $\mathrm{Ca}^{2+}$ ions anchor proteins on the membrane by directly contacting lipids. This model further indicates that $\mathrm{Ca}^{2+}$ is necessary for the GLA domain to bind to the membrane. Studies have shown that the GLA domain interacts with the negatively charged head group of the membrane phospholipid [199, 200]. More precisely, the phospholipid head group in the phospholipid (such as PtdSer) is bent so that the phosphate part of the phospholipid can form a coordination complex with the tightly bound $\mathrm{Ca}^{2+}$ in the GLA domain to performing their function. Furthermore, the stoichiometric ratio of the only complete PtdSer-GLA domain complex whose structure is determined by X-ray crystallography is 1:1, that in other words, one PtdSer molecule binding to one GLA domain [100].

Additionally, PROS1, one of the many coagulation factors consumed during the vigorous blood coagulation process, is a vitamin $\mathrm{K}$-dependent anticoagulant protein as well $[104,105]$. Its structure has been described in section TAM receptors family recognizes PtdSer in the immune and blood coagulation systems of this review. PROS1 is an essential inhibitory node in the coagulation reaction. In mice, congenital PROS1 deficiency can cause severe coagulopathy, leading to fatal coagulopathy and vascular dysplasia [106]. PROS1 can support the anticoagulant activity of APC. In human plasma, about $40 \%$ of PROS1 exists in the form of free protein S (FPS), while $60 \%$ of PROS1 forms a complex with C4b binding protein (C4BP). The complex causes the loss of the function of the PROS1 cofactor, and then C4BP can regulate the anticoagulant activity of APC [104]. PROS1 can also directly bind FXa and FVa to inhibit coagulation [25].

\section{The discoidin-like $\mathrm{C} 2$ domain of FV and FVIII binds to PtdSer}

The PtdSer recognition site of FVa and VIIIa is located in their N-terminal C2 domain [201, 202]. The light chain and lipid interactions of FVa and FVIIIa involve electrostatic and hydrophobic binding [203, 204]. Their light chains have the same domain homology, consisting of an A-type domain and two smaller C-type domains (Fig. 7). Their $\mathrm{C} 2$ domain is a $\beta$-barrel core with three relatively long loops (also called "spikes") protruding from one end $[205,206]$. Deleting the C2 domain from recombinant FV completely eliminates PtdSer-dependent binding, while mutants containing only the $\mathrm{C} 2$ domain (lack of $\mathrm{A} 3$ and C1 domains) can still bind to PtdSer [201]. The C2 domain of FVa contains a soluble phosphatidylserine (C6PS) binding pocket [207]. There is a pair of tryptophan residues (Trp ${ }^{2063}$ and $\operatorname{Trp}^{2064}$ ) in this lipid-binding pocket. Mutating these Trps eliminates the binding of FV to the membrane, indicating that these two Trp residues are necessary for the binding of FVa to PtdSer [208]. Some researchers have also shown that conservative mutations in the C2 domain of FVIII and FV can alter phospholipid binding and cofactor activity [209] and the $\operatorname{Trp}^{2313}-\mathrm{His}^{2315}$ fragment of the $\mathrm{C} 2$ domain of FVIII is involved in membrane binding [210]. All these indicate that the $\mathrm{C} 2$ domain of FVIII and FV is essential for PtdSer binding.

\section{Application of PtdSer in blood coagulation}

In recent years, with the development of the coagulation mechanism, more and more diseases, such as essential hypertension (EH) [5], Hemophilia A [6], can be detected or treated by the interaction between PtdSer and coagulation factors.

The PtdSer exposed on the surface of platelets facilitates the assembly of Tenase complex and Prothrombinase complex, thereby promoting the production of thrombin on the surface of activated platelets [103]. Furthermore, Emily C. Reddy et al. shows that procoagulant PtdSer-exposing platelets can be used as an antithrombotic target. Inhibition of procoagulant platelet formation may be an alternative method to reduce thrombosis without compromising hemostasis [213].

Thrombosis is the main cause of death in patients with EH [214]. Studies have found that inflammatory cytokines can enhance the procoagulant activity of platelets and endothelial cells through PtdSer exposure in patients with essential EH. Therefore, PtdSer blockade may be a feasible treatment strategy for such patients [5].

Hemophilia A is a genetic disease. It is a serious bleeding disorder caused by mutations in the FVIII (also known as anti-hemophilia factor A (AHF)) gene on the $\mathrm{X}$ chromosome [215]. Studies have shown that the decrease in PtdSer expression on the platelet surface measured by Annexin $\mathrm{V}$ binding indicates an increase in preventive bleeding in patients with severe FVIII deficiency [6].

Recent studies have also found that PtdSer, exposed on the surface of some mesenchymal stem cells and their derived extracellular vesicles, potentiates blood clot formation. While the use of annexin V to block PtdSer on their surface will lead to their procoagulant activity partially lost [216]. Also, studies have shown that PtdSer, exposed to blood cells and microparticles in patients 
with non-valvular atrial fibrillation, enables them procoagulant activity, which leads to thrombosis [217]. Therefore, further researches are needed to explore the disadvantage of the procoagulant effects which caused by PtdSer exposure in the treatment of mesenchymal stem cells and in some diseases.

\section{PtdSer binding recognition site in the immune and blood coagulation system}

Both GLA domain [82, 198, 218] and discoidin-like C2 domain [154, 205] can be used as PtdSer recognition binding sites during blood coagulation and immune processes. However, more PtdSer recognition and binding sites will be shared in the coagulation and immune process, which requires further experiment and research. It can be seen from the various recognition sites of PtdSer that some recognition sites (such as GLA domain) bind to the phosphate part of PtdSer [199, 200]. Therefore, not only PtdSer can interact with these domains, but PtdEtn and PA in phospholipids can also participate in the interaction with these domains in some processes (such as the recognition process with coagulation factors) $[219,220]$. PA performs better than PtdSer in the APC-dependent anticoagulation pathway. It may be due to the X group of PA has smaller steric hindrance than that of PtdSer, leading to the better entry of these phospholipids into the GLA domain and binding to $\mathrm{Ca}^{2+}$. However, the relatively large choline group of PtdCho will hinder the binding of the PtdCho and GLA domain [219].

Discoidin-like C2 domain not only appears in coagulation factors (FV and FVIII) $[201,202]$ but also in the ligand MFG-E8 of immune receptor $\alpha v \beta 3$ or $\alpha v \beta 5$ integrin [154, 155]. Moreover, the discoidin-like C2 domains of these proteins have similar structures, and they are all involved in the binding with PtdSer [221]. Occasionally, protein kinase $\mathrm{C}$ (PKC) in the nervous system also has a $\mathrm{PKC}-\mathrm{C} 2$ domain and participates in the binding of PtdSer in a $\mathrm{Ca}^{2+}$-dependent manner, thereby supports neuronal survival and differentiation in neuronal signal transduction [222, 223]. However, the PKC-C2 domain and discoidin-like $\mathrm{C} 2$ domain do not belong to the $\mathrm{C} 2$ domain though they are literally similar [224, 225].

There are IgV-like domains in some immune receptor proteins, like TIM family proteins [35], CD300 family proteins $[14,65,226]$, and RAGE [168]. The IgV-like domains of TIM-1, TIM-3, TIM-4, CD300a, and RAGE are all involved in the binding of PtdSer and are $\mathrm{Ca}^{2+}$ dependent. It could be speculated that whether other proteins containing IgV-like domains also have the property of binding to PtdSer, which needs further study.

Besides these Ptdser recognition sites mentioned above (Table 2), there are many other binding sites of PtdSer.
Annexin V can specifically recognize PtdSer, which needs the participation of $\mathrm{Ca}^{2+}$ [227]. Annexin $\mathrm{V}$ is widely used as a probe for detecting PtdSer on the cell surface [228-230]. In addition, TSP-1 repeats of BAI1 can bind to PtdSer in the extracellular area and promote the maximum phagocytosis of apoptotic cells through the ELMO-Dock180-Rac1 mechanism [66]. TSP-1 can also participate in many physiological and pathological processes, such as regulating PtdSer-dependent red blood cells in red blood cell-endothelial adhesion, which can be used as a potential therapeutic target [231]. Besides, the EGF-like domain is also a PtdSer recognition binding site. For example, Stabilin-1 and Stabilin-2 are binding with PtdSer through their EGF-like domain [67, $68]$ in endocytosis [164, 165], cell fusion, etc [166].

\section{PtdSer and PtdSer receptors may be involved in COVID-19}

Corona Virus Disease 2019 (COVID-19) is a viral infectious disease caused by Severe Acute Respiratory Syndrome Coronavirus 2 (SARS-CoV-2), which poses a major challenge to the World Health Organization [232]. Acute inflammation and coagulation abnormalities appear to be the main cause of death for thousands of patients worldwide [233, 234].

Studies have speculated that PtdSer exposure may be involved in the SARS-CoV-2 infection of the outer leaflets of the cell membrane. PtdSer may be a potential mechanism or participant of inflammation and coagulation abnormalities in COVID-19 patients [23, 24]. As mentioned above, PtdSer can be exposed on the surface of platelets during the coagulation process to promote the explosive production of thrombin to promote coagulation [28-32, 103]. PtdSer can also be exposed on the surface of apoptotic cells during the immune process as an "eat-me" signal, recognized by receptors on the surface of phagocytes (such as TAM or TIM). Then the apoptotic cells are engulfed by phagocytes, thereby avoiding inflammation in our body [19, 35, 47, 49]. However, under pathophysiological conditions, some viruses, such as HIV [8], EBOV [9-12], DENV [13-15], and RSV [16-18], can use the PtdSer exposed on their surface to bind to PtdSer receptors on immune cells to invade cells, thereby increasing the infectivity of these viruses. In addition, study [235] has shown that bacterial endotoxins can cause PtdSer exposure to excessively activate coagulation, leading to life-threatening disseminated intravascular coagulation (DIC).

Similarly, some researchers [236] have shown that the GAS6/PROS1/TAM system is assumed to participate in SARS-CoV-2 infection and complications. In section TAM receptors family recognizes PtdSer in the immune and blood coagulation systems of this review, it is mentioned that TAM participates in hemostasis and anti- 


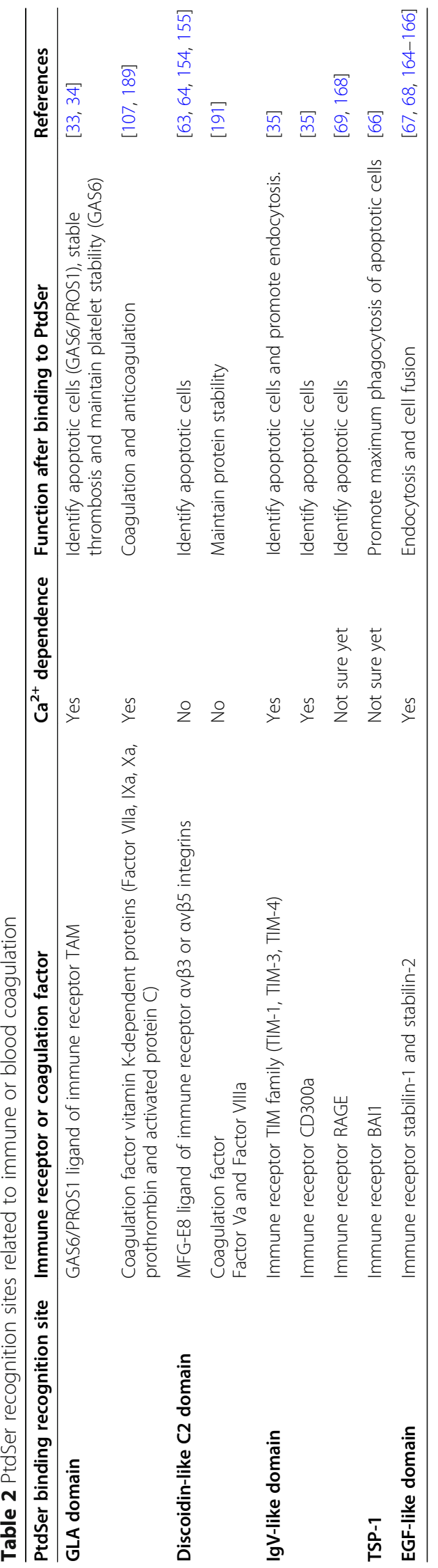


inflammatory $[25,80,81]$. GAS6/TAM can maintain thrombosis and platelet stability by promoting platelet aggregation $[25,33,81,101,102]$, and PtdSer is also involved in this process. PtdSer will be exposed to activated platelets not only to participate in the production of thrombin [103] but also to bind to GAS6 to activate TAM [33]. Data reveals that in patients with severe COVID-19, the level of soluble AXL in GAS6 and TAM will increase [237]. Study speculates that TAM signaling plays a vital role in the coagulopathy associated with excessive inflammation observed in COVID-19 [236]. In addition, because some viruses, such as Dengue [15], EBOV [10-12], and RSV [16-18], can infect the host through using the PtdSer exposed on their own surface to interact with the GAS6/ TAM of the host. Therefore, the study also predicts that GAS6, TAM, and PtdSer may participate in the invasion of the COVID-19 virus, which is an emerging and rapidly evolving situation [236].

Besides PtdSer, it was speculated that PROS1 might mechanically associate excessive blood coagulation with immune response in COVID-19 [238]. PROS1 can act as not only the activation ligand of TAM in the immune process [34, 83, 97-100], but also an anticoagulant in the blood coagulation process [104-106]. Furthermore, in these two processes, the two signal pathways cannot be activated without PtdSer [84, 107]. Study has shown that SARS-CoV-2 infection may be associated with explosive blood clotting [238]. The growing blood clots consume coagulation factors, including PROS1. Excessive consumption of PROS1 will not only cause abnormalities in the anticoagulation process but also unable the TAM receptors, especially MER, on immune cells to activate due to insufficient ligands. MER signaling will be silenced, resulting in inflammatory factors release of macrophages, such as IL-1 $\beta$, TNF- $\alpha$, etc. This study recommends testing the level of PROS1 during the standard blood test of COVID-19 patients. It shows that this measurement is beneficial for COVID-19 patients with coagulopathy and high D-dimer levels [238].

It has also been reported that the receptor for PtdSer, TIM-1 (KIM-1), may be the receptor for SARS-CoV-2 in the lung and kidney [239]. COVID-19 patients often have acute kidney injury [240], it has been demonstrated that TIM-1 is expressed in the epithelial cells of the lung and kidney of COVID-19 patients [239]. The enhanced expression of TIM-1 in human renal tubules increases the uptake of SARS-CoV-2 virions. It is also mentioned that many viruses, such as HIV [149], EBOV [9], DENV $^{12}$, can use the PtdSer exposed on their surface to bind to TIM to enter host cells. Therefore, we suggested that therapeutic targeting for TIM-1 can prevent and/or treat COVID-19 and prevent the above virus from infected.
Based on various studies [236, 239], it can be assumed that PtdSer may be exposed to SARS-CoV-2 and then infected the host through the TAM and/or TIM system, but there is no sufficient evidence to prove this hypothesis. In addition, studies have shown that the symptoms of COVID-19 are mostly acute inflammation and excessive blood clotting [233, 234]. As mentioned above, TAM has a significant effect on anti-inflammatory in the human body, which needs the participation of PtdSer $[34,80,241]$. PtdSer also plays a major role in the blood system, such as the formation of thrombin [28-32, 103], the stabilization of platelets, the maintenance of thrombosis by the GAS6/TAM system [25, 33, 81, 101, 102], and the anticoagulation process that depends on APC and PROS1 [104-106]. These coagulation [103] and anticoagulation [107] processes all have the participation of PtdSer. In summary, based on the perspective of the mechanism of PtdSer on blood coagulation, immunity, and viral infection, further study of the relationship between PtdSer and COVID-19 may lay a solid foundation for discovering COVID-19 infection mechanism and detection/treatment methods.

\section{Conclusion and perspective}

Immune systems and blood coagulation are vital for basic physiological functions. When tissues are injured, hemostasis is always the first step to control the injury. The hemostasis process usually requires platelets to complete. The cell-based coagulation mechanism has been explained in this article (section The Cell-Based Model of Hemostasis involving PtdSer). Blood coagulation and anticoagulation are very complicated processes involving the participation of various factors, and PtdSer is indispensable for activated platelets to produce thrombin [28-32, 103]. In addition, PtdSer plays a pivotal role in the $\mathrm{Ca}^{2+}$-dependent APC anticoagulant system [107, 196]. The lack of PtdSer on the surface of activated platelets will inevitably lead to a reduction in the production of thrombin, which will make the hemostasis process impossible to complete and cause a series of diseases. For example, Scott syndrome is a typical bleeding disorder caused by insufficient exposure of PtdSer in activated platelets [3].

Immune system is used to eliminate foreign antigens, or damaged cells, apoptotic cells, and tumor cells produced in our body to maintain homeostasis [242]. In this review, we mainly summarized the immune mechanism of macrophages to clear apoptotic cells responsible for innate immunity. Furthermore, PtdSer exposed to apoptotic cells is dominant for this innate immunity. For apoptotic cells to be successfully engulfed by macrophages, three conditions must be met: 
(i) PtdSer is exposed on the surface of apoptotic cells. The exposure of PtdSer requires the activation of scramblase Xkr8 and the inactivation of flippase ATP11C and ATP11A. Caspase3/7 participates in the activation and the inactivation [40-44].

(ii) PtdSer receptors on macrophages can recognize and bind PtdSer normally. There are many PtdSer receptors on macrophages, including TAM receptor family (TYRO3, AXL and MER) [34], TIM receptor family (TIM-1, TIM-3 and TIM-4, 35], $\alpha \mathrm{v} \beta 3$ or $\alpha v \beta 5$ integrins [63, 64], CD300a [14, 65], BAI1 [66], Stabilin [67, 68], RAGE [69], and LOX-1 [70], etc. Most of these receptors initiate endocytosis after recognized PtdSer on the surface of apoptotic cells. Moreover, TAM-mediated phagocytosis is the main mechanism to clear apoptotic cells [34].

(iii) PtdSer receptors can successfully activate downstream signal pathways after binding to PtdSer. For instance, the binding of TAM to PtdSer can activate Rac1 to activate phagocytosis [85] and inhibit the expression of inflammatory cytokine and NF-kB [19, 88]. It can also activate PI3K [25] and other signaling pathways. The recognition of TIM-1 and PtdSer can induce the activation, proliferation, and cytokine production of iNKT cells [146]. CD300b on macrophages can promote the phagocytosis of apoptotic cells through the DAP12-SykPI3K-AKT pathway after recognizing PtdSer [157]. After the PtdSer receptor BAIl binds to PtdSer, it acts synergistically through ELMO / Dock180 / Rac to promote the maximum phagocytosis of apoptotic cells [66]. Further researches have explained and hypothesized the signaling pathways of many receptors binding to PtdSer. Although there are still many mechanisms maintain unknown, all the research results point to that PtdSer is a key link in activating related signal pathways.

The coagulation process which PtdSer participated in can be briefly summarized as follows: (i) When tissue cells are injured, a little thrombin will be produced. (ii) A small amount of thrombin can activate platelets, and the $\mathrm{Ca}^{2+}$ level in the activated platelets increases, which leads to the activation of scramblase TMEM16F and the inactivation of flippase ATP11C and ATP11A, thereby causing PtdSer exposure on the platelet cell membrane. Then, PtdSer binding to various coagulation factors (such as FVIIIa, FVa, FIXa, and FXa) on the surface of activated platelets will promote their enzymatic activity and causes a burst of thrombin production. (iii) A large amount of thrombin can activate Fibrinogen (FI) and FXIII (FXIII) to generate Fibrin (FIa) and FXIIIa. FXIIIa can cross-link with FIa to form a Cross-linked fibrin clot, which is very important for the formation of a blood clot at the injured site. The formation of blood clots can prevent excessive bleeding [28-32, 103, 179].

The successful elimination of apoptotic cells is closely dependent on each of the above parts. Particular diseases will occur due to the absence of individual steps. For example, PtdSer exposure failure may be the cause of SLE [4]. Apoptotic cells progress to secondary necrosis integrity when they are not cleared in an efficient and timely manner, which will lead the phagocytes to release proinflammatory cytokines (i.e., TNF- $\alpha$, IL-1 $\beta$ ), thereby inducing inflammation [49].

Besides eliminating apoptotic cells, the binding of PtdSer to immune cell receptors can lead to other immune functions. For example, inhibiting the expression of inflammatory factors and NF- $\kappa B$ inhibit inflammation $[19,88]$. The recognition of PtdSer by TIM-1 can also induce iNKT cell activation, proliferation, and cytokine production [146].

However, some viruses (e.g. HIV [8], EBOV [9-12], DENV $[13,15])$ can use the intersection between PtdSer and PtdSer receptors to enter the host. These viruses induce the virus and invade the host by using the binding of PtdSer on the viral membrane and the TIM or TAM receptor on the host's immune cells. PtdSer on cancer cells can also bind to TIM-3 or TAM and promote immune escape [19-21].

Some factors and proteins in the process of coagulation and immunity have a similar structure (section PtdSer binding recognition site in the immune and blood coagulation system) that recognize and bind to PtdSer, such as GLA domain, discoidin-like C2 domain, IgV-like domain, etc. It is not excluded that other coagulation-related factors or immune receptors that have these PtdSer binding sites may also have the property of binding to PtdSer. More researches are needed and discovery to verify this hypothesis.

Since the coagulation and immune mechanisms have been continuously improved in recent years, many factors or receptors involved have been found. The relationship between the coagulation and immune mechanisms is being established $[25,26]$. This review summarized the role of PtdSer in blood coagulation and immunity. Some studies have speculated that TAM [236, 243] and PROS1 [238] may participate in the most urgent COVID-19 in a non-canonical way. The symptoms of COVID-19 patients are mostly acute inflammation and excessive blood clotting [233, 234]. Due to the role of PtdSer in coagulation and inflammation, it has been speculated that it may be a potential PtdSer-related mechanism or participant in COVID-19 inflammation and abnormal coagulation $[23,24]$. Studies $[236,239]$ have also assumed that PtdSer may be exposed to SARS-CoV-2 and then 
infected the host through the TAM and/or TIM system. Therefore, studying the intersection between PtdSer and COVID-19 may provide directions for discovering the mechanism of COVID-19 infection and detection/treatment methods.

PtdSer belongs to phospholipids on the membrane, and its importance for the biological membrane is obvious. PtdSer exposure is of great significance for blood coagulation [28-32, 103] and immunity [19], and PtdSer inside the cell is also indispensable to the nervous system [222]. Therefore, it is significant for the mechanism and treatment of concerning diseases to explore the mechanism and principles of PtdSer in blood coagulation and immunity.

\begin{abstract}
Abbreviations
AD: Alzheimer's disease; AHF: Anti-hemophilia factor A; AP-2: Adaptor protein complex 2; APC: Activated protein C; AT: Antithrombin; BAl1: Brain angiogenesis inhibitor 1; BSG: Basigin; C4BP: C4b binding protein; Caspase: Cysteine-containing aspartate-specific proteases; COVID-19: Corona Virus Disease 2019; Cryo-EM: Cryo-electron microscopy; DENV: Dengue virus; DIC: Disseminated intravascular coagulation; EBOV: Ebola virus: EGF: Epidermal growth factor; EH: Essential hypertension; Fl: Fibrinogen; Fla: Fibrin; Fll: Prothrombin; Flla: Thrombin; FIX: Factor IX; FN III: Fibronectinlike III; FPS: Free protein S; FV: Factor V; FVII: Factor VII; FVIII: Factor VIII; FX: Factor X; FXI: Factor XI; FXIII: Factor XIII; Gal-9: Galectin-9; GalCer: Galactosylceramide; GAS6: Growth-arrest-specific 6; GLA: Ycarboxyglutamate; HIV: Human Immunodeficiency Virus; HSPG: Heparan sulfate proteoglycans; IFN: Type I interferon; IgC-like: Immunoglobulin constant-like; Ig-like: Immunoglobulin-like; IgV-like: Immunoglobulin variablelike; IL: Interleukin; iNKT: Invariant natural killer T; ITIM: Immunoreceptor tyrosine-based inhibitory motif; KIM-1/TIM-1: Kidney-injury-molecule-1; LG: Laminin G; LOX-1: Lectin-like oxidized low-density lipoprotein receptor-1; MFG-E8: Milk fat globule epidermal growth factor 8; MILIBS: Metal iondependent ligand-binding site; NF-KB: Nuclear factor-KB; NPTN: Neuroplastin; PA: Phosphatidic acid; PtdCho: Phosphatidylcholine; PtdEtn: Phosphatidylethanolamine; PI3K: Phosphatidylinositol 3-kinases; PKC: Protein kinase C; PROS1: Protein S; PtdSer: Phosphatidylserine; pSVRPLL: di-leucine sequence; PTK: Protein tyrosine kinase; RAGE: Receptor for advanced glycation end products; RGD: Arg-Gly-Asp; Rho: Ras Homologue; RPE: Retinal pigment epithelium; RSV: Respiratory Syncytial Virus; RTKs: Receptor protein tyrosine kinase; SARS-CoV-2: Severe Acute Respiratory Syndrome Coronavirus 2; SHBG: Sex hormone binding globulin;

SLE: Systemic lupus erythematosus; SM: Sphingomyelin; SOCS: Suppressor of cytokine signaling; TAM: Receptor Tyrosine kinases TYRO3, AXL and MER; Tc1: T cytotoxic cell; TF: Tissue factor; TFPI: Tissue factor pathway inhibitor; Th1: T helper 1; TIM: T cell/transmembrane, immunoglobulin and mucin; TLR: Toll-like receptor; TM: Transmembrane domain;

TMEM16F: Transmembrane protein 16F; TNF: Tumor necrosis factor; TSP-

1: Thrombospondin-1; vWF: von Willebrand Factor; Xkr8: Xk-Related Protein8
\end{abstract}

\section{Acknowledgments}

We would thank Xuantin Liu and other laboratory members for ongoing discussion and manuscript proofreading. We would apologize to authors whose relevant research was not cited due to the space limitation.

\section{Authors' contributions}

All authors reviewed the literature and wrote first drafts of respective sections. JW, CY and JZ integrated the sections and form the final version of the manuscript. All authors read and approved the final manuscript.

\section{Funding}

This work was sponsored by Basic Reasearch Program of Shanghai (20JC1412200), Shanghai Municipal Science and Technology Major Project (2020YFA0113000, 2018YFA0109800), Key Laboratory of Computational Neuroscience and Brain-Inspired Intelligence (LCNBI) and ZJLab.
Availability of data and materials

Not applicable.

\section{Declarations}

Ethics approval and consent to participate

Not applicable.

\section{Consent for publication}

All authors consent to publication.

\section{Competing interests}

The authors declare no competing interests.

\section{Author details}

${ }^{1}$ School of Life Sciences, Shanghai University, 99 Shangda Road, Shanghai 200444, China. ${ }^{2}$ Institute of Basic Medical Sciences, Chinese Academy of Medical Sciences, School of Basic Medicine, Peking Union Medical College, No. 5 Dongdansantiao, Beijing 100005, China. ${ }^{3}$ Centre of Excellence in Tissue Engineering, Chinese Academy of Medical Sciences, Beijing, China. ${ }^{4}$ Beijing Key Laboratory of New Drug Development and Clinical Trial of Stem Cell Therapy (BZ0381), Beijing, China.

Received: 30 September 2021 Accepted: 1 November 2021

Published online: 15 January 2022

References

1. Devaux PF, Morris R. Transmembrane asymmetry and lateral domains in biological membranes. Traffic. 2004;5(4):241-6.

2. Gascard P, et al. Asymmetric distribution of phosphoinositides and phosphatidic acid in the human erythrocyte membrane. Biochim Biophys Acta. 1991;1069(1):27-36.

3. Wielders $\mathrm{SJH}$, et al. Absence of platelet-dependent fibrin formation in a patient with Scott syndrome. Throm Haemost. 2009;102(07):76-82.

4. Kawano M, Nagata S. Lupus-like autoimmune disease caused by a lack of Xkr8, a caspase-dependent phospholipid scramblase. Proc Natl Acad Sci. 2018;115(9):2132-7.

5. Li J, et al. Inflammatory cytokines enhance procoagulant activity of platelets and endothelial cells through phosphatidylserine exposure in patients with essential hypertension. J Thromb Thrombolysis. 2020:1-8.

6. Croteau SE, et al. Decreased platelet surface phosphatidylserine predicts increased bleeding in patients with severe factor VIII deficiency. J Thromb Haemost: JTH. 2021.

7. Neher JJ, et al. Inhibition of microglial phagocytosis is sufficient to prevent inflammatory neuronal death. J Immunol. 2011;186(8):4973-83.

8. Yasen A, et al. HIV internalization into oral and genital epithelial cells by endocytosis and macropinocytosis leads to viral sequestration in the vesicles. Virology. 2018;515:92-107.

9. Rhein BA, et al. Characterization of human and murine T-cell immunoglobulin mucin domain 4 (TIM-4) IgV domain residues critical for Ebola virus entry. J Virol. 2016;90(13):6097-111.

10. Bhattacharyya $S$, et al. Enveloped viruses disable innate immune responses in dendritic cells by direct activation of TAM receptors. Cell Host Microbe. 2013;14(2):136-47.

11. Shimojima M, et al. Tyro3 family-mediated cell entry of Ebola and Marburg viruses. J Virol. 2006;80(20):10109-16.

12. Shimojima M, Ikeda Y, Kawaoka Y. The mechanism of Axl-mediated Ebola virus infection. J Infect Dis. 2007;196(Suppl 2):S259-63.

13. Chu L-W, et al. TIM-1 as a signal receptor triggers dengue virus-induced autophagy. Int J Mol Sci. 2019;20(19):4893.

14. Carnec $X$, et al. The phosphatidylserine and phosphatidylethanolamine receptor CD300a binds dengue virus and enhances infection. J Virol. 2016; 90(1):92-102.

15. Meertens $L$, et al. The TIM and TAM families of phosphatidylserine receptors mediate dengue virus entry. Cell Host Microbe. 2012;12(4):544-57.

16. Shibata $\mathrm{T}$, et al. Axl receptor blockade ameliorates pulmonary pathology resulting from primary viral infection and viral exacerbation of asthma. J Immunol. 2014;192(8):3569-81.

17. Shibata $T$, et al. Respiratory syncytial virus infection exacerbates pneumococcal pneumonia via Gas6/Axl-mediated macrophage polarization. J Clin Invest. 2020;130(6):3021-37. 
18. Shibata T, Ato M. A critical role of Gas6/Axl signal in allergic airway responses during RSV vaccine-enhanced disease. Immunol Cell Biol. 2017; 95(10):906-15.

19. Birge RB, et al. Phosphatidylserine is a global immunosuppressive signal in efferocytosis, infectious disease, and cancer. Cell Death Differ. 2016;23(6): 962-78.

20. Yin $Y$, et al. Phosphatidylserine-targeting antibody induces M1 macrophage polarization and promotes myeloid-derived suppressor cell differentiation. Cancer Immunol Res. 2013;1(4):256-68.

21. Park M, Kang KW. Phosphatidylserine receptor-targeting therapies for the treatment of cancer. Arch Pharm Res. 2019;42(7):617-28.

22. Stanford JC, et al. Efferocytosis produces a prometastatic landscape during postpartum mammary gland involution. J Clin Inv. 2014;124(11):4737-52.

23. Argañaraz GA, da Fonseca Palmeira J, Argañaraz ER. Phosphatidylserine inside out: a possible underlying mechanism in the inflammation and coagulation abnormalities of COVID-19. Cell Commun Signal. 2020;18(1):1-10.

24. Lind SE. Phosphatidylserine is an overlooked mediator of COVID-19 thromboinflammation. Heliyon. 2021:e06033.

25. van der Meer JHM, van der Poll T, van 't Veer C. TAM receptors, Gas6, and protein S: roles in inflammation and hemostasis. Blood, J Am Soc Hematol. 2014:123(16):2460-9.

26. Verhamme P, Hoylaerts MF. Hemostasis and inflammation: two of a kind. Thromb J. 2009:7(1):1-3.

27. Kim S-J, Davis RP, Jenne CN. Platelets as modulators of inflammation. Thieme Med Publishers. 2018.

28. Hoffman M. A cell-based model of coagulation and the role of factor Vlla. Blood Rev. 2003;17:S1-5.

29. Hoffman M, Monroe lii DM. A cell-based model of hemostasis. Thromb Haemost. 2001;85(06):958-65

30. Smith SA. The cell-based model of coagulation. J Vet Emerg Crit Care. 2009; 19(1):3-10.

31. Sucker C, Zotz RB. The cell-based coagulation model, in Perioperative hemostasis: Springer; 2015. p. 3-11.

32. Stoilova-McPhie, S., Factor VIII and Factor V Membrane Bound Complexes, in Macromolecular Protein Complexes III: Structure and Function. 2021, Springer.153-175.

33. Cosemans J, et al. Potentiating role of Gas6 and Tyro3, Axl and Mer (TAM) receptors in human and murine platelet activation and thrombus stabilization. J Thromb Haemost. 2010;8(8):1797-808.

34. Lemke G, Rothlin CV. Immunobiology of the TAM receptors. Nat Rev Immunol. 2008;8(5):327-36.

35. Freeman GJ, et al. TIM genes: a family of cell surface phosphatidylserine receptors that regulate innate and adaptive immunity. Immunol Rev. 2010; 235(1):172-89.

36. Kerr JFR, Wyllie AH, Currie AR. Apoptosis: a basic biological phenomenon with wideranging implications in tissue kinetics. $\mathrm{Br}$ J Cancer. 1972;26(4):239-57.

37. Orrenius S. Mitochondrial regulation of apoptotic cell death. Toxicol Lett. 2004;149(1-3):19-23.

38. Locksley RM, Killeen N, Lenardo MJ. The TNF and TNF receptor superfamilies: integrating mammalian biology. Cell. 2001;104(4):487-501.

39. Igney $\mathrm{FH}, \mathrm{Krammer} \mathrm{PH}$. Death and anti-death: tumour resistance to apoptosis. Nat Rev Cancer. 2002;2(4):277-88.

40. Suzuki J, et al. Xk-related protein 8 and CED-8 promote phosphatidylserine exposure in apoptotic cells. Science. 2013;341(6144):403-6.

41. Segawa K, Kurata S, Nagata S. Human type IV P-type ATPases that work as plasma membrane phospholipid Flippases and their regulation by caspase and calcium. J Biol Chem. 2016;291(2):762-72.

42. Segawa K, et al. Caspase-mediated cleavage of phospholipid flippase for apoptotic phosphatidylserine exposure. Science. 2014;344(6188):1164-8.

43. Suzuki J, Imanishi E, Nagata S. Xkr8 phospholipid scrambling complex in apoptotic phosphatidylserine exposure. Proc Natl Acad Sci. 2016;113(34) 9509-14.

44. Nagata $\mathrm{S}$, et al. Exposure of phosphatidylserine on the cell surface. Cell Death Differ. 2016;23(6):952-61

45. Riedl SJ, Shi Y. Molecular mechanisms of caspase regulation during apoptosis. Nat Rev Mol cell Biol. 2004;5(11):897-907.

46. Budihardjo I, et al. Biochemical pathways of caspase activation during apoptosis. Annual Rev Cell Dev Biol. 1999;15(1):269-90.

47. Graham DK, et al. The TAM family: phosphatidylserine sensing receptor tyrosine kinases gone awry in cancer. Nat Rev Cancer. 2014;14(12):769-85.
48. Das M, Zhu C, Kuchroo VK. Tim-3 and its role in regulating anti-tumor immunity. Immunol Rev. 2017;276(1):97-111.

49. Kimani SG, et al. Contribution of defective PS recognition and Efferocytosis to chronic inflammation and autoimmunity. Front Immunol. 2014:5:566.

50. Suzuki J, et al. Calcium-dependent phospholipid scrambling by TMEM16F. Nature. 2010;468(7325):834-8.

51. Bull $L N$, et al. A gene encoding a P-type ATPase mutated in two forms of hereditary cholestasis. Nat Genet. 1998;18(3):219-24.

52. Onat $\mathrm{OE}$, et al. Missense mutation in the ATPase, aminophospholipid transporter protein ATP8A2 is associated with cerebellar atrophy and quadrupedal locomotion. Eur J Hum Genet. 2013;21(3):281-5.

53. Siggs OM, et al. The P4-type ATPase ATP11C is essential for B lymphopoiesis in adult bone marrow. Nat Immun. 2011:12(5):434-40.

54. Yabas $M$, et al. ATP11C is critical for the internalization of phosphatidylserine and differentiation of B lymphocytes. Nat Immun. 2011;12(5):441-9.

55. Andersen JP, et al. P4-ATPases as phospholipid flippases-structure function, and enigmas. Front Physiol. 2016;7:275

56. Nagata S, Sakuragi T, Segawa K. Flippase and scramblase for phosphatidylserine exposure. Curr Opin Immunol. 2020;62:31-8.

57. Kansal S, et al. Development of nanocapsules bearing doxorubicin for macrophage targeting through the phosphatidylserine ligand: a system for intervention in visceral leishmaniasis. J Antimicrob Chemother. 2012;67(11): 2650-60.

58. Brouckaert $\mathrm{G}$, et al. Phagocytosis of necrotic cells by macrophages is phosphatidylserine dependent and does not induce inflammatory cytokine production. Mol Biol Cell. 2004;15(3):1089-100.

59. Hiraizumi M, et al. Cryo-EM structures capture the transport cycle of the P4ATPase flippase. Science. 2019;365(6458):1149-55.

60. Tsokos GC. Autoimmunity and organ damage in systemic lupus erythematosus. Nat Immunol. 2020;21(6):605-14.

61. Fadok VA, et al. Exposure of phosphatidylserine on the surface of apoptotic lymphocytes triggers specific recognition and removal by macrophages. J Immunol. 1992;148(7):2207-16.

62. Gardai SJ, et al. Recognition ligands on apoptotic cells: a perspective. J leukocyte Biol. 2006:79(5):896-903.

63. Kaminska A, Enguita FJ, Stepien EL. Lactadherin: an unappreciated haemostasis regulator and potential therapeutic agent. Vascul Pharmacol. 2018;101:21-8.

64. Hanayama $\mathrm{R}$, et al. Autoimmune disease and impaired uptake of apoptotic cells in MFG-E8-deficient mice. Science. 2004;304(5674):1147-50.

65. Simhadri VR, et al. Human CD300a binds to phosphatidylethanolamine and phosphatidylserine, and modulates the phagocytosis of dead cells. Blood, J Am Soc Hematol. 2012;119(12):2799-809.

66. Park D, et al. BAl1 is an engulfment receptor for apoptotic cells upstream of the ELMO/Dock180/Rac module. Nature. 2007;450(7168):430-4

67. Park S-Y, et al. Epidermal growth factor-like domain repeat of stabilin-2 recognizes phosphatidylserine during cell corpse clearance. Mol Cell Biol. 2008:28(17):5288-98.

68. Park S-Y, Kim I-S. Stabilin receptors: role as phosphatidylserine receptors. Biomolecules. 2019:9(8):387.

69. He M, et al. Receptor for advanced glycation end products binds to phosphatidylserine and assists in the clearance of apoptotic cells. EMBO Rep. 2011;12(4):358-64

70. Murphy JE, et al. LOX-1 scavenger receptor mediates calcium-dependent recognition of phosphatidylserine and apoptotic cells. Biochem J. 2006; 393(1):107-15.

71. Lemke G. Biology of the TAM receptors. Cold Spring Harb Perspect Biol. 2013;5(11):a009076.

72. Hubbard SR, Till JH. Protein tyrosine kinase structure and function. Annual Rev Biochem. 2000;69(1):373-98.

73. Zwick E, Bange J, Ullrich A. Receptor tyrosine kinase signalling as a target for cancer intervention strategies. Endocrine-related Cancer. 2001; 8(3):161-73.

74. Seitz HM, et al. Macrophages and dendritic cells use different AxI/Mertk Tyro3 receptors in clearance of apoptotic cells. J Immunol. 2007:178(9): 5635-42.

75. Rothlin CV, et al. TAM receptors are pleiotropic inhibitors of the innate immune response. Cell. 2007;131(6):1124-36.

76. Caraux A et al. Natural killer cell differentiation driven by Tyro3 receptor tyrosine kinases. Nat Immunol. 2006;7(7):747-54. 
77. Prieto $A L$, Weber $J L$, Lai $C$. Expression of the receptor protein-tyrosine kinases Tyro-3, Axl, and Mer in the developing rat central nervous system. J Comp Neurol. 2000;425(2):295-314.

78. Prasad D, et al. TAM receptor function in the retinal pigment epithelium. Mol Cell Neurosci. 2006;33(1):96-108.

79. Wang $\mathrm{H}$, et al. Immunoexpression of tyro 3 family receptors - tyro 3, Ax and Mer-and their ligand Gas6 in postnatal developing mouse testis. J Histochem Cytochem. 2005;53(11):1355-64.

80. Lee $\mathrm{C}-\mathrm{H}$, Chun T. Anti-inflammatory role of TAM family of receptor tyrosine kinases via modulating macrophage function. Mol Cells. 2019;42(1):1

81. Angelillo-Scherrer A, et al. Role of Gas6 in erythropoiesis and anemia in mice. J Clin Invest. 2008;118(2):583-96.

82. Stitt TN, et al. The anticoagulation factor protein $\mathrm{S}$ and its relative, Gas6, are ligands for the tyro 3/Axl family of receptor tyrosine kinases. Cell. 1995;80(4): 661-70.

83. Mark MR, et al. Characterization of Gas6, a member of the superfamily of G domain-containing proteins, as a ligand for Rse and Axl. J Biol Chem. 1996; 271(16):9785-9.

84. Lew ED, et al. Differential TAM receptor-ligand-phospholipid interactions delimit differential TAM bioactivities. Elife. 2014;3:e03385.

85. Wu Y, et al. A role for Mer tyrosine kinase in av $\beta 5$ integrin-mediated phagocytosis of apoptotic cells. J Cell Sci. 2005;118(3):539-53.

86. Wu Y, Tibrewal N, Birge RB. Phosphatidylserine recognition by phagocytes: a view to a kill. Trends Cell Biol. 2006;16(4):189-97.

87. Castellano F, Montcourrier P, Chavrier P. Membrane recruitment of Rac1 triggers phagocytosis. J Cell Sci. 2000;113(17):2955-61.

88. Cvetanovic M, Ucker DS. Innate immune discrimination of apoptotic cells: repression of proinflammatory macrophage transcription is coupled directly to specific recognition. J Immunol. 2004;172(2):880-9.

89. Deng T, et al. Toll-like receptor-mediated inhibition of Gas6 and ProS expression facilitates inflammatory cytokine production in mouse macrophages. Immunology. 2012;135(1):40-50.

90. Tibrewal N, et al. Autophosphorylation docking site Tyr-867 in Mer receptor tyrosine kinase allows for dissociation of multiple signaling pathways for phagocytosis of apoptotic cells and down-modulation of lipopolysaccharide-inducible NF-kB transcriptional activation. J Biol Chem. 2008;283(6):3618-27

91. Tourdot BE, et al. Immunoreceptor tyrosine-based inhibitory motif (ITIM)mediated inhibitory signaling is regulated by sequential phosphorylation mediated by distinct nonreceptor tyrosine kinases: a case study involving PECAM-1. Biochemistry. 2013;52(15):2597-608.

92. Graham DK, et al. Cloning and mRNA expression analysis of a novel human protooncogene, c-mer. Cell Growth Differ: Mol Biol J Am Assoc Cancer Res. 1994;5(6):647-57.

93. O'Bryan JP, et al. Axl, a transforming gene isolated from primary human myeloid leukemia cells, encodes a novel receptor tyrosine kinase. Mol Cell Biol. 1991;11(10):5016-31.

94. Lai C, Gore M, Lemke G. Structure, expression, and activity of tyro 3, a neural adhesion-related receptor tyrosine kinase. Oncogene. 1994;9(9): 2567-78.

95. Nagata K, et al. Identification of the product of growth arrest-specific gene 6 as a common ligand for Axl, sky, and Mer receptor tyrosine kinases. J Biol Chem. 1996:271(47):30022-7.

96. Hafizi S, Dahlbäck B. Gas6 and protein S. FEBS J. 2006;273(23):5231-44

97. Segawa K, Nagata S. An apoptotic 'eat me'signal: phosphatidylserine exposure. Trends Cell Biol. 2015;25(11):639-50.

98. Lemke G. Phosphatidylserine is the signal for TAM receptors and their ligands. Trends Biochem Sci. 2017;42(9):738-48.

99. Lemke G, Burstyn-Cohen T. TAM receptors and the clearance of apoptotic cells. Ann N Y Acad Sci. 2010;1209:23-9.

100. Huang $M$, et al. Structural basis of membrane binding by Gla domains of vitamin K-dependent proteins. Nat Struct Mol Biol. 2003;10(9):751-6.

101. Angelillo-Scherrer A, et al. Role of Gas6 receptors in platelet signaling during thrombus stabilization and implications for antithrombotic therapy. J Clin Investig. 2005;115(2):237-46.

102. Law LA, et al. GAS6/TAM pathway signaling in hemostasis and thrombosis. Front Med. 2018:5:137.

103. Robert FA, Zwaal PC, Bevers EM. Lipid-protein interactions in blood coagulation. Biochimica et Biophysica Acta (BBA). 1998
104. Rezende SM, Simmonds RE, Lane DA. Coagulation, inflammation, and apoptosis: different roles for protein $\mathrm{S}$ and the protein S-C4b binding protein complex. Blood. 2004;103(4):1192-201.

105. Castoldi E, Hackeng TM. Regulation of coagulation by protein S. Curr Opin Hematol. 2008;15(5):529-36.

106. Burstyn-Cohen T, Heeb MJ, Lemke G. Lack of protein S in mice causes embryonic lethal coagulopathy and vascular dysgenesis. J Clin Invest. 2009; 119(10):2942-53.

107. Tans $\mathrm{G}$, et al. Comparison of anticoagulant and procoagulant activities of stimulated platelets and platelet-derived microparticles. Blood. 1991;77(12): 2641-8.

108. Hong S, et al. Complement and microglia mediate early synapse loss in Alzheimer mouse models. Science. 2016:352(6286):712-6.

109. Nonaka S, Nakanishi H. Microglial clearance of focal apoptotic synapses. Neurosci Lett. 2019:707:134317.

110. Ran S, Downes A, Thorpe PE. Increased exposure of anionic phospholipids on the surface of tumor blood vessels. Cancer Res. 2002;62(21):6132-40.

111. Sharma B, Kanwar SS. Phosphatidylserine: a cancer cell targeting biomarker. Semin Cancer Biol. 2018:52(Pt 1):17-25.

112. Ran S, Thorpe PE. Phosphatidylserine is a marker of tumor vasculature and a potential target for cancer imaging and therapy. Int J Radiat Oncol Biol Phys. 2002;54(5):1479-84.

113. Hutterer $M$, et al. Axl and growth arrest-specific gene 6 are frequently overexpressed in human gliomas and predict poor prognosis in patients with glioblastoma multiforme. Clin Cancer Res. 2008;14(1):130-8.

114. Sun WS, Fujimoto J, Tamaya T. Coexpression of growth arrest-specific gene 6 and receptor tyrosine kinases Axl and sky in human uterine endometrial cancers. Ann Oncol. 2003;14(6):898-906.

115. Loges $S$, et al. Malignant cells fuel tumor growth by educating infiltrating leukocytes to produce the mitogen Gas6. Blood. 2010;115(11):2264-73.

116. Abboud-Jarrous $\mathrm{G}$, et al. Protein S drives oral squamous cell carcinoma tumorigenicity through regulation of AXL. Oncotarget. 2017;8(8):139864002

117. Sadahiro $H$, et al. Activation of the receptor tyrosine kinase $A X L$ regulates the immune microenvironment in glioblastoma. Cancer Res. 2018;78(11): 3002-13.

118. Kasikara C, et al. Phosphatidylserine sensing by TAM receptors regulates AKT-dependent Chemoresistance and PD-L1 expression. Mol Cancer Res. 2017;15(6):753-64

119. Ghosh Roy S. TAM receptors: a phosphatidylserine receptor family and its implications in viral infections. Int Rev Cell Mol Biol. 2020;357:81-122.

120. Rodriguez-Manzanet $R$, et al. The costimulatory role of TIM molecules. Immunol Rev. 2009;229(1):259-70.

121. Su EW, Lin JY, Kane LP. TIM-1 and TIM-3 proteins in immune regulation. Cytokine. 2008;44(1):9-13.

122. Meyers $\mathrm{JH}$, et al. TIM-4 is the ligand for TIM-1, and the TIM-1-TIM-4 interaction regulates T cell proliferation. Nat Immunol. 2005;6(5):455-64.

123. Khademi $M$, et al. T cell Ig-and mucin-domain-containing molecule-3 (TIM-3) and TIM-1 molecules are differentially expressed on human Th1 and Th2 cells and in cerebrospinal fluid-derived mononuclear cells in multiple sclerosis. J Immunol. 2004:172(11):7169-76.

124. Anderson $A C$, et al. Promotion of tissue inflammation by the immune receptor Tim-3 expressed on innate immune cells. Science. 2007;318(5853): 1141-3.

125. Frisancho-Kiss $S$, et al. Cutting edge: T cell Ig mucin-3 reduces inflammatory heart disease by increasing CTLA-4 during innate immunity. J Immunol. 2006;176(11):6411-5.

126. Oikawa T, et al. Preferential involvement of Tim-3 in the regulation of hepatic CD8+ T cells in murine acute graft-versus-host disease. J Immunol. 2006:177(7):4281-7.

127. Kobayashi N, et al. TIM-1 and TIM-4 glycoproteins bind phosphatidylserine and mediate uptake of apoptotic cells. Immunity. 2007;27(6):927-40.

128. Zhang X, et al. TIM-4 is expressed on invariant NKT cells but dispensable for their development and function. Oncotarget. 2016;7(44):71099.

129. Zhang Q, et al. TIM-4 promotes the growth of non-small-cell lung cancer in a RGD motif-dependent manner. Br J Cancer. 2015;113(10):1484-92.

130. Ruoslahti E. RGD and other recognition sequences for integrins. Annual Rev Cell Dev Biol. 1996:12(1):697-715.

131. Santiago C, et al. Structures of T cell immunoglobulin mucin receptors 1 and 2 reveal mechanisms for regulation of immune responses by the TIM receptor family. Immunity. 2007;26(3):299-310. 
132. DeKruyff RH, et al. T cell/transmembrane, Ig, and mucin-3 allelic variants differentially recognize phosphatidylserine and mediate phagocytosis of apoptotic cells. J Immunol. 2010;184(4):1918-30.

133. Santiago C, et al. Structures of T cell immunoglobulin mucin protein 4 show a metal-ion-dependent ligand binding site where phosphatidylserine binds. Immunity. 2007;27(6):941-51.

134. Miyanishi M, et al. Identification of Tim4 as a phosphatidylserine receptor. Nature. 2007:450(7168):435-9.

135. Zhu C, et al. The Tim-3 ligand galectin-9 negatively regulates T helper type 1 immunity. Nat Immunol. 2005;6(12):1245-52.

136. Nakae S, et al. Phenotypic differences between Th1 and Th17 cells and negative regulation of Th1 cell differentiation by IL-17. J Leukocyte Biol. 2007;81(5):1258-68.

137. Nakayama M, et al. Tim-3 mediates phagocytosis of apoptotic cells and cross-presentation. Blood. 2009;113(16):3821-30.

138. Zhu C, Anderson AC, Kuchroo VK. TIM-3 and its regulatory role in immune responses, in Negative Co-Receptors and Ligands: Springer; 2010. p. 1-15.

139. Lee J, et al. Phosphotyrosine-dependent coupling of Tim-3 to T-cell receptor signaling pathways. Mol Cell Biol. 2011;31(19):3963-74.

140. Zeidan AM, Komrokji RS, Brunner AM. TIM-3 pathway dysregulation and targeting in cancer. Expert Rev Anticancer Ther. 2021

141. Yanagihashi $Y$, et al. Mouse macrophages show different requirements for phosphatidylserine receptor Tim4 in efferocytosis. Proc Natl Acad Sci U S A. 2017;114(33):8800

142. Moon B, et al. Mertk interacts with Tim-4 to enhance Tim-4-mediated efferocytosis. Cells. 2020;9(7):1625

143. Zakharova L, Svetlova M, Fomina AF. T cell exosomes induce cholesterol accumulation in human monocytes via phosphatidylserine receptor. J Cell Physiol. 2007;212(1):174-81.

144. Klibi J, et al. Blood diffusion and Th1-suppressive effects of galectin-9containing exosomes released by Epstein-Barr virus-infected nasopharyngeal carcinoma cells. Blood. 2009;113(9):1957-66.

145. Théry C, Ostrowski M, Segura E. Membrane vesicles as conveyors of immune responses. Nat Rev Immunol. 2009;9(8):581-93.

146. Lee $\mathrm{H}-\mathrm{H}$, et al. Apoptotic cells activate NKT cells through T cell Ig-like mucinlike-1 resulting in airway hyperreactivity. J Immunol. 2010;185(9):5225-35.

147. Ichimura $T$, et al. Kidney injury molecule-1 is a phosphatidylserine receptor that confers a phagocytic phenotype on epithelial cells. J Clin Investig. 2008;118(5):1657-68

148. Rees AJ, Kain R. Kim-1/Tim-1: from biomarker to therapeutic target. Nephrol Dial Transplant. 2008;23(11):3394-6

149. Hazenberg MD, et al. Persistent immune activation in HIV-1 infection is associated with progression to AIDS. Aids. 2003;17(13):1881-8.

150. Li M, et al. TIM-family proteins inhibit HIV-1 release. Proc Natl Acad Sci. 2014; 111(35):E3699-707.

151. Cohen PL, Shao W-H. Gas6/TAM receptors in systemic lupus erythematosus. Dis Markers. 2019:2019.

152. Pagani S, et al. New insights into the role of Tyro3, Axl, and Mer receptors in rheumatoid arthritis. Dis Markers. 2020;2020

153. Finnemann SC, Rodriguez-Boulan E. Macrophage and retinal pigment epithelium phagocytosis: apoptotic cells and photoreceptors compete for av $\beta 3$ and av $\beta 5$ integrins, and protein kinase $C$ regulates av $\beta 5$ binding and cytoskeletal linkage. J Exp Med. 1999;190(6):861-74.

154. Ye $\mathrm{H}$, et al. NMR solution structure of $\mathrm{C} 2$ domain of MFG-E8 and insights into its molecular recognition with phosphatidylserine. Biochimica et Biophysica Acta (BBA)-Biomembranes. 2013;1828(3):1083-93.

155. Li $\mathrm{H}_{\text {, et }}$ al. The Structure and Properties of MFG-E8 and the In vitro Assessment of Its Toxic Effects on Myoblast Cells. Protein Expr Purif. 2020: 105720.

156. Ushikubo M, et al. Milk fat globule epidermal growth factor 8 (MFG-E8) on monocytes is a novel biomarker of disease activity in systemic lupus erythematosus. Lupus. 2021.

157. Murakami Y, et al. CD300b regulates the phagocytosis of apoptotic cells via phosphatidylserine recognition. Cell Death Differ. 2014;21(11):1746-57.

158. Choi S-C, et al. Cutting edge: mouse CD300f (CMRF-35-like molecule-1) recognizes outer membrane-exposed phosphatidylserine and can promote phagocytosis. J Immunol. 2011;187(7):3483-7.

159. Dimitrova $M$, et al. CD300c is uniquely expressed on CD56 bright natural kille cells and differs from CD300a upon ligand recognition. Sci Rep. 2016;6:23942.

160. Vitallé J, et al. CD300 receptor family in viral infections. Eur J Immunol. 2019. 49(3):364-74.
161. Borrego F. The CD300 molecules: an emerging family of regulators of the immune system. Blood. 2013;121(11):1951-60.

162. Das $\mathrm{S}$, et al. Brain angiogenesis inhibitor 1 is expressed by gastric phagocytes during infection with helicobacter pylori and mediates the recognition and engulfment of human apoptotic gastric epithelial cells. FASEB J. 2014;28(5):2214-24.

163. Zhu D, et al. BAl1 suppresses medulloblastoma formation by protecting p53 from Mdm2-mediated degradation. Cancer cell. 2018;33(6):1004-1016. e5.

164. Park SY, et al. Rapid cell corpse clearance by stabilin-2, a membrane phosphatidylserine receptor. Cell Death Diff. 2008;15(1):192-201.

165. Park S-Y, et al. Stabilin-1 mediates phosphatidylserine-dependent clearance of cell corpses in alternatively activated macrophages. J Cell Sci. 2009; 122(18):3365-73.

166. Park S-Y, et al. Stabilin-2 modulates the efficiency of myoblast fusion during myogenic differentiation and muscle regeneration. Nat Commun. 2016;7(1): $1-15$

167. Lee $W$, et al. Macrophagic Stabilin-1 restored disruption of vascular integrity caused by Sepsis. Thromb Haemost. 2018;118(10):1776-89.

168. Teissier T, Boulanger É. The receptor for advanced glycation end-products (RAGE) is an important pattern recognition receptor (PRR) for inflammaging. Biogerontology. 2019;20(3):279-301.

169. Liu Z, et al. Activation of RAGE-dependent endoplasmic reticulum stress associates with exacerbated post-myocardial infarction ventricular arrhythmias in diabetes. Am J Physiol-Endocrinol Metab.

170. Schröter D, Höhn A. Role of advanced glycation end products in carcinogenesis and their therapeutic implications. Curr Pharm Design. 2018; 24(44):5245-51.

171. Paudel YN, et al. Impact of HMGB1, RAGE, and TLR4 in Alzheimer's disease (AD): from risk factors to therapeutic targeting. Cells. 2020;9(2):383.

172. Kerkeni M, Gharbi J. RAGE receptor: may be a potential inflammatory mediator for SARS-COV-2 infection. Med Hypotheses. 2020.

173. Moriwaki H, et al. Ligand specificity of LOX-1, a novel endothelial receptor for oxidized low density lipoprotein. Arterioscler Thromb Vasc Biol. 1998; 18(10):1541-7.

174. Sagar D, et al. LOX-1: a potential driver of cardiovascular risk in SLE patients. PloS ONE. 2020;15(3):e0229184

175. Murdocca M, et al. LOX-1 and cancer: an indissoluble liaison. Cancer Gene Ther:1-11.

176. Zwaal RF. Membrane and lipid involvement in blood coagulation. Biochim Biophys Acta. 1978:515(2):163-205.

177. Schroit AJ, Zwaal RF. Transbilayer movement of phospholipids in red cell and platelet membranes. Biochim Biophys Acta. 1991;1071(3):313-29.

178. Zwaal RFA, Schroit AJ. Pathophysiologic implications of membrane phospholipid asymmetry in blood cells. Blood, J Am Soc Hematol. 1997; 89(4):1121-32.

179. Bevers EM, Comfurius P, Zwaal RF. Changes in membrane phospholipid distribution during platelet activation. Biochim Biophys Acta. 1983;736(1):57-66

180. Kunzelmann K, et al. Molecular functions of anoctamin 6 (TMEM16F): chloride channel, cation channel, or phospholipid scramblase? Pflügers Archiv-Eur J Physiol. 2014;466(3):407-14.

181. Scudieri $P$, et al. Ion channel and lipid scramblase activity associated with expression of TMEM16F/ANO6 isoforms. J Physiol. 2015;593(17):3829-48.

182. Le T, et al. An inner activation gate controls TMEM16F phospholipid scrambling. Nat Commun. 2019;10(1):1-13.

183. Zwaal RFA, Comfurius P, Bevers EM. Scott syndrome, a bleeding disorder caused by defective scrambling of membrane phospholipids. Biochimica et Biophysica Acta (BBA)-Mol Cell Biol Lipids. 2004;1636(2-3):119-28.

184. Hussain JF, Mahaut-Smith MP. Reversible and irreversible intracellular Ca2+ spiking in single isolated human platelets. J Physiol. 1999;514(Pt 3):713-8.

185. Takatsu H, et al. Phospholipid flippase ATP11C is endocytosed and downregulated following ca $2+-$ mediated protein kinase $\mathrm{C}$ activation. Nat Commun. 2017:8(1):1-15.

186. McMahon HT, Boucrot E. Molecular mechanism and physiological functions of clathrin-mediated endocytosis. Nat Rev Mol Cell Biol. 2011;12(8):517.

187. Kelly BT, et al. A structural explanation for the binding of endocytic dileucine motifs by the AP2 complex. Nature. 2008;456(7224):976-9.

188. Pitcher C, et al. Cluster of differentiation antigen 4 (CD4) endocytosis and adaptor complex binding require activation of the CD4 endocytosis signal by serine phosphorylation. Mol Biol Cell. 1999;10(3):677-91. 
189. Nelsestuen GL. Role of gamma-carboxyglutamic acid. An unusual protein transition required for the calcium-dependent binding of prothrombin to phospholipid. J Biol Chem. 1976;251(18):5648-56.

190. Davie EW, Fujikawa K, Kisiel W. The coagulation cascade: initiation, maintenance, and regulation. Biochemistry. 1991;30(43):10363-70.

191. Kane WH, Davie EW. Blood coagulation factors V and VIII: structural and functional similarities and their relationship to hemorrhagic and thrombotic disorders; 1988.

192. Stoilova-McPhie $S$, et al. Domain organization of membrane-bound factor VIII. Biopolymers Original Res Biomol. 2013;99(7):448-59.

193. Broze GJ Jr. Tissue factor pathway inhibitor and the revised theory of coagulation. Annu Rev Med. 1995;46:103-12.

194. van Boven HH, Lane DA. Antithrombin and its inherited deficiency states. Semin Hematol. 1997;34(3):188-204.

195. Lindahl U, Kjellén L. Heparin or Heparan sulfate-what is the difference. Thromb Haemost. 1991;65(01):044-8.

196. Dahlbäck B, Stenflo J. The molecular basis of blood disease; 2000

197. Esmon CT. Molecular events that control the protein $C$ anticoagulant pathway. Thromb Haemost. 1993;69(01):029-35.

198. Vermeer C. $\mathrm{Y}$-Carboxyglutamate-containing proteins and the vitamin Kdependent carboxylase. Biochem J. 1990;266(3):625-36

199. Ohkubo YZ, Tajkhorshid E. Distinct structural and adhesive roles of Ca2+ in membrane binding of blood coagulation factors. Structure. 2008;16(1):72-81.

200. Tavoosi N, et al. Molecular determinants of phospholipid synergy in blood clotting. J Biol Chem. 2011;286(26):23247-53.

201. Ortel TL, et al. Deletion analysis of recombinant human factor $V$. evidence for a phosphatidylserine binding site in the second C-type domain. J Biol Chem. 1992;267(6):4189-98.

202. Gilbert GE, Baleja JD. Membrane-binding peptide from the C2 domain of factor VIII forms an amphipathic structure as determined by NMR spectroscopy. Biochemistry. 1995;34(9):3022-31.

203. Kalafatis M, Jenny RJ, Mann KG. Identification and characterization of a phospholipid-binding site of bovine factor Va. J Biol Chem. 1990;265(35): 21580-9.

204. Lecompte MF, Bouix G, Mann KG. Electrostatic and hydrophobic interactions are involved in factor $V a$ binding to membranes containing acidic phospholipids. J Biol Chem. 1994;269(3):1905-10.

205. Macedo-Ribeiro S, et al. Crystal structures of the membrane-binding C2 domain of human coagulation factor V. Nature. 1999;402(6760):434-9.

206. Pratt KP, et al. Structure of the C2 domain of human factor VIII at $1.5 \AA$ resolution. Nature. 1999;402(6760):439-42.

207. Srivastava A, et al. Soluble phosphatidylserine binds to a single identified site in the C2 domain of human factor Va. Biochemistry. 2001;40(28):8246-55

208. Majumder $\mathrm{R}$, et al. The phosphatidylserine binding site of the factor Va C2 domain accounts for membrane binding but does not contribute to the assembly or activity of a human factor Xa- factor Va complex. Biochemistry. 2005;44(2):711-8.

209. Gilbert GE, et al. Conservative mutations in the C2 domains of factor VIII and factor $V$ alter phospholipid binding and cofactor activity. Blood, J Am Soc Hematol. 2012;120(9):1923-32.

210. Liu Z, et al. Trp2313-His2315 of factor VIII C2 domain is involved in membrane binding STRUCTURE OF a COMPLEX BETWEEN THE C2 DOMAIN AND AN INHIBITOR OF MEMBRANE BINDING. J Biol Chem. 2010;285(12): 8824-9.

211. Dahlbäck B. Human coagluation factor $\vee$ purification and thrombincatalyzed activation. J Clin Inv. 1980;66(3):583-91.

212. Kalafatis M, Rand MD, Mann KG. Factor Va-membrane interaction is mediated by two regions located on the light chain of the cofactor. Biochemistry. 1994;33(2):486-93.

213. Reddy EC, Rand ML. Procoagulant phosphatidylserine-exposing platelets in vitro and in vivo. Front Cardiovasc Med. 2020;7.

214. Nadar S, Lip GYH. The prothrombotic state in hypertension and the effects of antihypertensive treatment. Curr Pharm Design. 2003;9(21):1715-32

215. Antonarakis SE, Kazazian HH, Tuddenham EGD. Molecular etiology of factor VIII deficiency in hemophilia a. Hum mutat. 1995;5(1):1-22.

216. Silachev DN, et al. Effect of MSCs and MSC-Derived Extracellular Vesicles on Human Blood Coagulation. Cells. 2019;8(3).

217. Wang $L$, et al. Phosphatidylserine-exposing blood cells and microparticles induce procoagulant activity in non-valvular atrial fibrillation. Int J Cardiol. 2018;258:138-43
218. Nakano T, et al. Cell adhesion to phosphatidylserine mediated by a product of growth arrest-specific gene 6. J Biol Chem. 1997;272(47):29411-4.

219. Tavoosi N, et al. Factor VII and protein C are phosphatidic acid-binding proteins. Biochemistry. 2013;52(33):5545-52.

220. Medfisch SM, et al. Phosphatidylethanolamine-phosphatidylserine binding synergy of seven coagulation factors revealed using Nanodisc arrays on silicon photonic sensors. Sci Rep. 2020;10(1):1-7.

221. Novakovic VA, et al. Membrane-binding properties of the factor VIII C2 domain. Biochem J. 2011:435(1):187-96.

222. Kim H-Y, Huang BX, Spector AA. Phosphatidylserine in the brain: metabolism and function. Prog Lipid Res. 2014;56:1-18.

223. Verdaguer $\mathrm{N}$, et al. Ca2+ bridges the $\mathrm{C} 2$ membrane-binding domain of protein kinase Ca directly to phosphatidylserine. EMBO J. 1999;18(22):6329-38.

224. Leventis PA, Grinstein S. The distribution and function of phosphatidylserine in cellular membranes. Annua Rev Biophysics. 2010;39:407-27.

225. Lemmon MA. Membrane recognition by phospholipid-binding domains. Nat Rev Mol Cell Biol. 2008;9(2):99-111.

226. Clark GJ, et al. The CD300 family of molecules are evolutionarily significant regulators of leukocyte functions. Trends Immunol. 2009;30(5):209-17.

227. Reutelingsperger CPM, Van Heerde WL, Annexin V. The regulator of phosphatidylserine-catalyzed inflammation and coagulation during apoptosis. Cell Mol Life Sci CMLS. 1997:53(6):527-32

228. Tait JF, Gibson D. Phospholipid binding of annexin V: effects of calcium and membrane phosphatidylserine content. Arc Biochem Biophys. 1992;298(1): 187-91.

229. Kay JG, et al. Phosphatidylserine dynamics in cellular membranes. Mol Biol Cell. 2012;23(11):2198-212.

230. Koopman, G., et al., Annexin $\vee$ for flow cytometric detection of phosphatidylserine expression on B cells undergoing apoptosis. 1994.

231. Betal SG, Setty BNY. Phosphatidylserine-positive erythrocytes bind to immobilized and soluble thrombospondin-1 via its heparin-binding domain. Transl Res. 2008;152(4):165-77.

232. Dashraath $P$, et al. Coronavirus disease 2019 (COVID-19) pandemic and pregnancy. Am J obstet Gynecol. 2020;222(6):521-31.

233. Xu Z, et al. Pathological findings of COVID-19 associated with acute respiratory distress syndrome. Lancet Respir Med. 2020;8(4):420-2.

234. Connors JM, Levy JH. COVID-19 and its implications for thrombosis and anticoagulation. Blood, J Am Soc Hematol. 2020;135(23):2033-40.

235. Yang $X$, et al. Bacterial endotoxin activates the coagulation cascade through gasdermin D-dependent phosphatidylserine exposure. Immunity. 2019;51(6): 983-996. e6.

236. Tutusaus A, et al. Role of vitamin K-dependent factors protein S and GAS6 and TAM receptors in SARS-CoV-2 infection and COVID-19-associated Immunothrombosis. Cells. 2020;9(10):2186.

237. Huckriede, J., et al., Markers of NETosis and DAMPs are altered in critically ill COVID-19 patients. 2020

238. Lemke G, Silverman GJ. Blood clots and TAM receptor signalling in COVID19 pathogenesis. Nat Rev Immunol. 2020:1-2.

239. Ichimura T, et al. KIM-1/TIM-1 is a Receptor for SARS-CoV-2 in Lung and Kidney. medRxiv. 2020

240. Rubin S, et al. Characterisation of acute kidney injury in critically ill patients with severe coronavirus disease-2019 (COVID-19). medRxiv. 2020

241. Rothlin CV, et al. TAM receptor signaling in immune homeostasis. Annu Rev Immunol. 2015;33:355-91.

242. Parham P. The immune system. Garland Sci. 2014

243. Wang $S$, et al. AXL is a candidate receptor for SARS-CoV-2 that promotes infection of pulmonary and bronchial epithelial cells. Cell Res. 2021:1-15.

\section{Publisher's Note}

Springer Nature remains neutral with regard to jurisdictional claims in published maps and institutional affiliations. 